\title{
Efeitos do Pneumoperitônio sobre a Hemodinâmica e Função Renais de Cães Ventilados com Volume e Pressão Controlados *
}

\section{Effects of Pneumoperitoneum on Renal Hemodynamics and Function of Dogs under Volume and Pressure-Controlled Ventilation}

\author{
Armando Vieira de Almeida, $T_{S A}{ }^{1}$; Eliana Marisa Ganem, TSA ${ }^{2}$
}

\section{RESUMO}

Almeida AV, Ganem EM - Efeitos do Pneumoperitônio sobre a Hemodinâmica e Função Renais de Cães Ventilados com Volume e Pressão Controlados

JUSTIFICATIVA E OBJETIVOS: Não existem estudos que associem os efeitos determinados pelas modalidades ventilatórias às repercussões renais durante o pneumoperitônio. O objetivo deste trabalho foi avaliar as alterações na hemodinâmica e função renais determinadas pelo pneumoperitônio em cães com ventilação a volume e pressão controlados.

MÉTODO: Dezesseis cães anestesiados com tiopental sódico e fentanil foram divididos em Grupo 1, volume controlado e Grupo 2, pressão controlada e submetidos a pneumoperitônio de 10 e $15 \mathrm{mmHg}$. Foram estudados fluxo sangüíneo renal, resistência vascular renal, depuração de para-aminohipurato de sódio, sódio plasmático, potássio plasmático, osmolalidade plasmática, depuração de creatinina, fração de filtração, volume urinário, osmolalidade urinária, depuração osmolar, depuração de água livre, depuração de sódio, excreção urinária de sódio, excreção fracionária de sódio, depuração de potássio, excreção urinária de potássio, excreção fracionária de potássio. Os dados foram coletados em 4 momentos. $M_{1}$, antes do pneumoperitônio; $M_{2}, 30$ minutos após pneumoperitônio com $10 \mathrm{mmHg} ; M_{3}, 30$ minutos após pneumoperitônio com 15 $\mathrm{mmHg} ; \mathrm{M}_{4}, 30$ minutos após a deflação do pneumoperitônio.

RESULTADOS: As depurações de para-aminohipurato de sódio e creatinina permaneceram constantes em ambos os grupos durante o experimento. Os valores plasmáticos do sódio e do potássio não se alteraram. Ocorreu diminuição a partir de $M_{2}$ da depuração e da excreção fracionária de potássio em ambos os grupos.

CONCLUSÕES: As modalidades ventilatórias não determinaram diferenças na hemodinâmica renal entre os grupos estudados. O pneumoperitônio, ocasionando

* Recebido do (Received from) Laboratório Experimental do CET/SBA do Departamento de Anestesiologia da Faculdade de Medicina de Botucatu (FMB - UNESP), para o Programa de Pós-Graduação em Anestesiologia, Mestrado

1. Pós-Graduando (Doutorado) do Programa de Pós-Graduação em Anestesiologia da FMB - UNESP. Bolsista CAPES

2. Professora Adjunta Livre-Docente do CET/SBA do Departamento de

Anestesiologia da Faculdade de Medicina de Botucatu - UNESP

Apresentado (Submitted) em 28 de maio de 2003

Aceito (Accepted) para publicação em 25 de agosto de 2003

Endereço para correspondência (Correspondence to)

Dr. Armando Vieira de Almeida

Rua Padre João Crippa, 3299/204 Bairro São Francisco

79010-180 Campo Grande, MS

(C) Sociedade Brasileira de Anestesiologia, 2004 compressão do parênquima renal, pode ter determinado alterações na reabsorção e/ou secreção do potássio.

Unitermos: ANIMAL: cão; CIRURGIA, Abdominal: videolaparoscópica; VENTILAÇÃO: controlada mecânica

\section{SUMMARY}

Effects of Pneumoperitoneum on Renal Hemodynamics and Function of Dogs under Volume and Pressure-Controlled Ventilation

BACKGROUND AND OBJECTIVES: There are no studies associating ventilatory mode effects on renal repercussions during pneumoperitoneum. This study aimed at evaluating pneumoperitoneum-induced renal hemodynamics and function changes in dogs under volume and pressure controlled ventilation.

METHODS: This study involved 16 dogs anesthetized with sodium thiopental and fentanyl, which were divided in two groups: Group 1: volume controlled; and Group 2: pressure controlled, both submitted to 10 and $15 \mathrm{mmHg}$ pneumoperitoneum. The following parameters were evaluated: renal blood flow, renal vascular resistance, sodium para-aminohippurate clearance, plasma sodium, plasma potassium, plasma osmolality, creatinine clearance, filtration fraction, urinary volume, urinary clearance, osmolar clearance, free water clearance, sodium clearance, sodium urinary excretion, sodium fractional excretion, potassium clearance, potassium urinary excretion and potassium fractional excretion. Data were collected in 4 moments: $M_{1}$ before pneumoperitoneum, $M_{2}, 30$ minutes after $10 \mathrm{mmHg}$ pneumoperitoneum, $M_{3}, 30$ minutes after $15 \mathrm{mmHg}$ pneumoperitoneum, $M_{4}, 30$ minutes after pneumoperitoneum deflation.

RESULTS: Sodium para-aminohippurate and creatinine clearance remained constant for both groups throughout the experiment. Plasma sodium and potassium were not changed. There has been potassium clearance and fractional excretion decrease as from $M_{2}$ in both groups.

CONCLUSIONS: Ventilatory modes have not promoted renal hemodynamic differences between groups. Pneumoperitoneum, by compressing renal parenchyma, may have determined changes in potassium reabsorption and/or secretion.

Key Words: ANIMAL: dog; SURGERY, Abdominal: videolaparoscopic; VENTILATION: mechanical controlled

\section{INTRODUÇÃO}

Nas cirurgias videolaparoscópicas, o aumento da pressão abdominal determinado pelo pneumoperitônio, assim como o posicionamento do paciente no intra-operatório, exercem influências nos diversos órgãos e sistemas. 
Com relação aos rins, a compressão do parênquima, das artérias e das veias renais durante o pneumoperitônio culmina com oligúria ${ }^{1}$. Quando a pressão intra-abdominal aumenta de 0 para $20 \mathrm{mmHg}$, a resistência vascular renal aumenta em $555 \%$ e o ritmo de filtração glomerular diminui em $25 \%{ }^{2}$. As alterações renais também estão associadas à ativação do sistema neuroendócrino ${ }^{3,4}$. Foram observadas concentrações plasmáticas aumentadas de vasopressina ${ }^{3-5}$, de renina 4,6-9 e de endotelina ${ }^{10}$ durante o pneumoperitônio. O aumento destas substâncias no plasma induz à vasoconstrição e contribui para elevações da resistência vascular sistêmica.

A ativação do sistema renina-angiotensina-aldosterona leva à vasoconstrição renal mediada pela angiotensina II, promovendo desvio do fluxo sangüíneo da medula para a córtex renal, piorando a perfusão do órgão ${ }^{8}$.

Quando se institui a ventilação mecânica, as variações na pressão do interior da caixa torácica alteram a pré e a pós-carga, o que pode comprometer a hemodinâmica cardiovascular ${ }^{11}$ e a hemodinâmica e função renais, especialmente nos pacientes graves. A estas alterações, vem-se somar o aumento da pressão intra-abdominal (PIA) que, dependendo das condições clínicas e posicionamento do paciente, determina importantes mudanças nos parâmetros ventilatórios estabelecidos, o que, conseqüentemente, pode determinar variações hemodinâmicas.

Aoligúria é um fenômeno freqüente durante o pneumoperitônio, evidenciando alteração na homeostase renal de etiologia não elucidada. Este estudo teve como objetivo determinar a existência de correlação entre duas modalidades ventilatórias, volume e pressão-controlados, e as alterações da hemodinâmica e função renais em cães submetidos a pneumoperitônio de 10 e 15 mmHg.

\section{MÉTODO}

Após a aprovação do Comitê de Ética em Experimentação Animal, foram utilizados 16 cães adultos, de ambos os sexos, sem raça definida, cujos pesos variaram de 15 a 23 quilogramas, fornecidos pelo Biotério do Campus de Botucatu da Universidade Estadual Paulista Júlio de Mesquita Filho.

$\mathrm{Na}$ seleção dos animais foram excluídos aqueles que não apresentavam aspecto sadio. Os 16 cães foram distribuídos em dois grupos experimentais, obedecendo ao critério de sorteio.

Foram criados dois grupos de estudo com 8 animais em cada um. Os grupos diferenciaram-se pela modalidade ventilatória utilizada, ou seja:

Grupo 1 (G1) - Ventilação com volume controlado; Grupo 2 (G2) - Ventilação com pressão controlada.

Após período de jejum de 12 horas com livre acesso à água, os animais foram pesados e anestesiados com injeção venosa de pentobarbital sódico (15 mg. $\left.\mathrm{kg}^{-1}\right)$, e fentanil (15 $\mu \mathrm{g} . \mathrm{kg}^{-1}$ ), colocados em decúbito dorsal horizontal sobre a goteira de Claude Bernard, quando foi efetuada a medida, em centímetros, da extremidade do nariz ao ânus, pela superfí- cie ventral. Utilizando-se esta distância e o peso em quilograma, foi estimada a superfície corporal por meio de tabelas de dados fisiológicos normais.

Foi realizada, a seguir, intubação traqueal com sonda número 38 provida de balonete de baixa pressão e elevada complacência, para instalar ventilação mecânica dos pulmões. Os animais foram ventilados com oxigênio a $100 \%$. Os do Grupo 1, cuja ventilação foi volume controlado, pelo apareIho de anestesia da Ohmeda, monitorizado pelo aparelho $\mathrm{AS} / 3$ da Datex-Engstrom, receberam volume corrente suficiente para manter a $\mathrm{P}_{\mathrm{ET}} \mathrm{CO}_{2}$ (pressão expiratória final de $\mathrm{CO}_{2}$ ) entre 35 e $45 \mathrm{mmHg}$. Os animais do Grupo 2, cuja ventilação foi com pressão controlada, pelo aparelho de anestesia da Ohmeda, monitorizado pelo aparelho AS/3 da Datex-Engstrom, foram ventilados com a pressão suficiente para manter $\mathrm{P}_{\mathrm{ET}} \mathrm{CO}_{2}$ entre 35 e $45 \mathrm{mmHg}$. Amensuração da $\mathrm{P}_{\mathrm{ET}} \mathrm{CO}_{2}$ foi realizada pela coleta da amostra do ar expirado junto à peça em $\mathrm{Y}$ do circuito respiratório. Em ambos os grupos, a freqüência respiratória foi fixada inicialmente em 10 movimentos por minuto. Para facilitar a ventilação artificial, foi administrado brometo de pancurônio na dose de 0,07 $\mathrm{mg} \cdot \mathrm{kg}^{-1}$ como dose inicial, e de $0,008 \mathrm{mg} \cdot \mathrm{kg}^{-1}$, como dose complementar.

Foi realizada dissecção das veias femorais direita e esquerda e das artérias femorais direita e esquerda, as quais foram cateterizadas com cateter de polietileno, introduzido de 2 a 3 $\mathrm{cm}$. No cateter da veia femoral esquerda foi administrada solução de Ringer na dose de $6 \mathrm{ml} \cdot \mathrm{kg}^{-1} \cdot \mathrm{h}^{-1}$ com infusão controlada por meio de bomba microprocessada "Anne" ${ }^{\circledR}$ Abbott Laboratórios (USA), e infusão contínua de tiopental sódico $\left(100 \mu \mathrm{g} \cdot \mathrm{kg}^{-1} \cdot \mathrm{min}^{-1}\right)$ e fentanil $\left(0,1 \mu \mathrm{g} \cdot \mathrm{kg}^{-1} \cdot \mathrm{min}^{-1}\right)$.

Trinta minutos após o início da infusão de solução de Ringer, foi administrado por via venosa o prime de creatinina (30 $\mathrm{mg} \cdot \mathrm{kg}^{-1}$ ) e para-aminohipurato de sódio (PAH), $4 \mathrm{mg} \cdot \mathrm{kg}^{-1}$. Foi instalada, a seguir, até o término da experiência, a infusão contínua da solução de creatinina $(0,6 \mathrm{~g} \%)$ e PAH $(0,24$ g\%) em solução de Ringer, sendo administrados, por minuto, 0,6 mg de creatinina e $0,24 \mathrm{mg}$ de PAH por quilograma de peso do animal, com infusão controlada por meio de bomba microprocessada "Anne".

A extremidade do cateter da artéria femoral esquerda foi conectada ao módulo de leitura de pressão arterial do aparelho AS/3 da Datex-Engstron. O cateter da artéria femoral direita foi utilizado para colheita de sangue arterial.

Após tricotomia da região cervical direita, foi realizada dissecção da veia jugular externa direita para passagem do cateter de Swan-Ganz conforme técnica descrita por Gouvea e col. ${ }^{12}$.

O acesso à cavidade peritoneal foi obtido com auxílio de incisão cirúrgica, de aproximadamente 5 centímetros de extensão, em região mediana da parede abdominal, por meio da qual foi introduzido, sob visão direta, cateter de polietileno $12 \mathrm{G}$, seguindo-se sutura dos planos cirúrgicos. A extremidade do cateter foi conectada ao aparelho insuflador, sendo então realizado o pneumoperitônio com o $\mathrm{CO}_{2}$. As pressões intra-abdominais de $10 \mathrm{mmHg}$ e $15 \mathrm{mmHg}$ foram alcançadas e mantidas constantes pelo módulo de controle de pressão e Vol. 54, N 3, Maio - Junho, 2004 
fluxo do aparelho. A desinsuflação da cavidade peritoneal ocorreu após a desconexão entre o cateter e o aparelho insuflador seguida de pequena compressão da cavidade abdominal.

O cateterismo uretral foi realizado com sonda de polivinil e o esvaziamento vesical foi obtido com leve compressão suprapúbica.

Para a realização dos períodos de depuração (30 minutos de duração) a urina foi coletada em provetas graduadas. Na metade do período foram colhidos, em tubos de centrífuga com heparina, $5 \mathrm{ml}$ de sangue venoso.

Os atributos renais, fluxo sangüíneo renal, resistência vascular renal, depuração de $\mathrm{PAH}$, sódio plasmático, potássio plasmático, osmolalidade plasmática, depuração de creatinina, fração de filtração, volume urinário, osmolalidade urinária, depuração osmolar, depuração de água livre, depuração de sódio, excreção urinária de sódio, excreção fracionária de sódio, depuração de potássio, excreção urinária de potássio e excreção fracionária de potássio foram obtidos nos seguintes momentos após a administração do prime de PAH e de creatinina:

$\mathrm{M}_{1}$ - 60 minutos após a administração do prime de PAH e de creatinina e imediatamente antes do pneumoperitônio com pressão de $10 \mathrm{mmHg}$;

$\mathrm{M}_{2}$ - 90 minutos após a administração do prime de PAH e de creatinina, 30 minutos após o pneumoperitônio de 10 $\mathrm{mmHg}$ e imediatamente antes do pneumoperitônio com pressão de $15 \mathrm{mmHg}$;

$M_{3}-120$ minutos após a administração do prime de PAH e de creatinina, 30 minutos após o pneumoperitônio de 15 mmHg e imediatamente antes da deflação do pneumoperitônio;

$\mathrm{M}_{4}$ - 150 minutos após a administração do prime de PAH e de creatinina, 30 minutos após a deflação do pneumoperitônio e imediatamente antes do sacrifício do animal.

Para a realização da dosagem de vasopressina foram colhidos $5 \mathrm{ml}$ de sangue venoso, em tubos de centrífuga refrigerados e com heparina, nos seguintes momentos:

$\mathrm{M}_{1}{ }^{\prime}$ - Imediatamente antes do pneumoperitônio com pressão de $10 \mathrm{mmHg}$;

$\mathrm{M}_{2}$ - 15 minutos após o estabelecimento do pneumoperitônio com pressão de $10 \mathrm{mmHg}$;

$\mathrm{M}_{3}{ }^{\prime}-15$ minutos após o estabelecimento do pneumoperitônio com pressão de $15 \mathrm{mmHg}$;

$\mathrm{M}_{4}$ ' - 15 minutos após a deflação do pneumoperitônio.

A dosagem de vasopressina foi realizada pela técnica de radioimunoensaio, utilizando-se o Kit DSL-1800 Arginine Vasopressin Radioimmunoassay.

Terminado o experimento, os animais foram sacrificados com injeção venosa de $10 \mathrm{ml}$ de cloreto de potássio a 19,1\%.

Para o tratamento estatístico, foi utilizada a Análise de Perfil para o teste das hipóteses Morrison ${ }^{13}$. Em todas as hipóte- ses testadas, as estatísticas $\mathrm{F}$ calculadas foram consideradas significativas quando $p<0,05$.

\section{RESULTADOS}

A análise estatística dos valores referentes à depuração de para-aminohipurato de sódio (Figura 1) e do fluxo sangüíneo renal (Figura 2) mostrou que os dois grupos apresentaram perfis similares, não havendo diferença entre os momentos no decorrer do experimento.

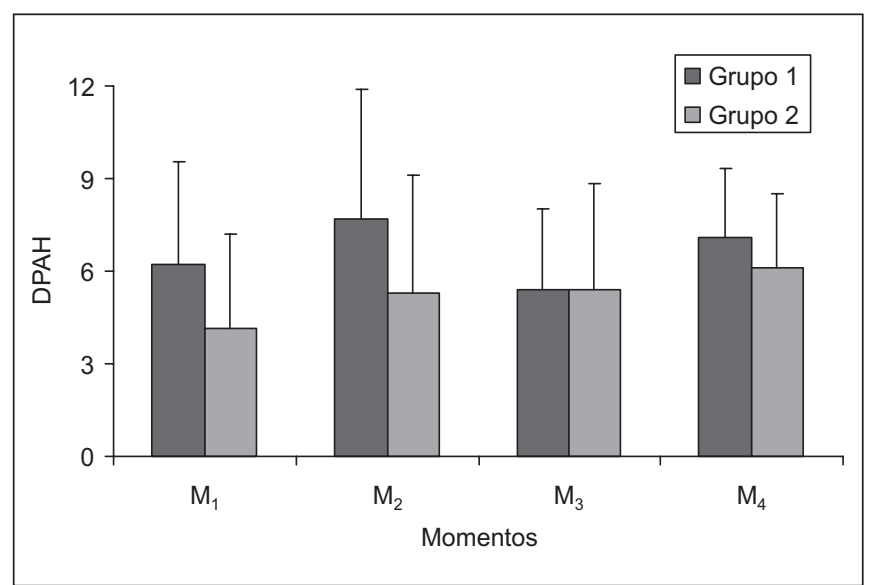

Figura 1 - Depuração de Para-Aminohipurato de Sódio. Média e Desvio Padrão dos Valores Observados nos Dois Grupos Experimentais

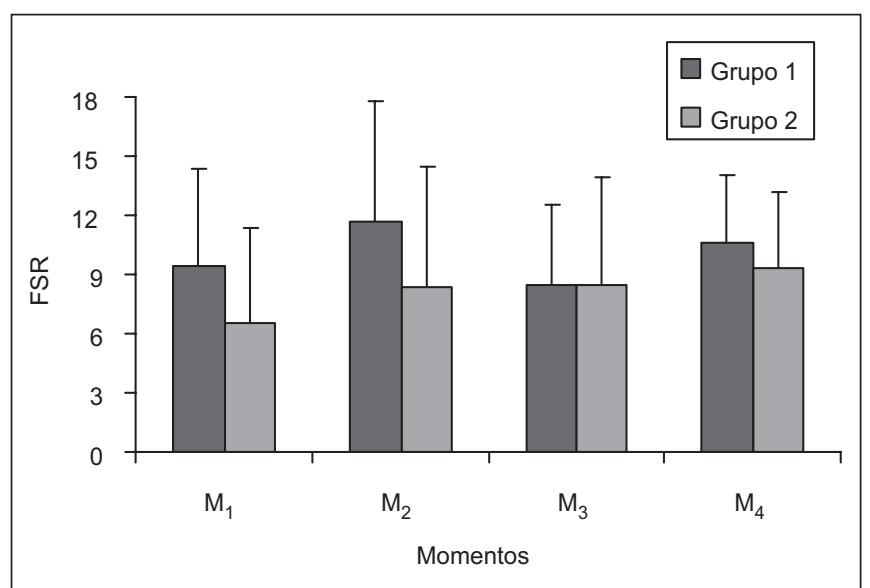

Figura 2 - Fluxo Sangüíneo Renal. Média e Desvio Padrão dos Valores Observados nos Dois Grupos Experimentais

Já a análise estatística dos valores referentes à resistência vascular renal mostrou que eles foram significativamente menores no Grupo 1, porém não houve diferença entre os momentos no transcorrer do experimento em ambos os grupos (Figura 3). 


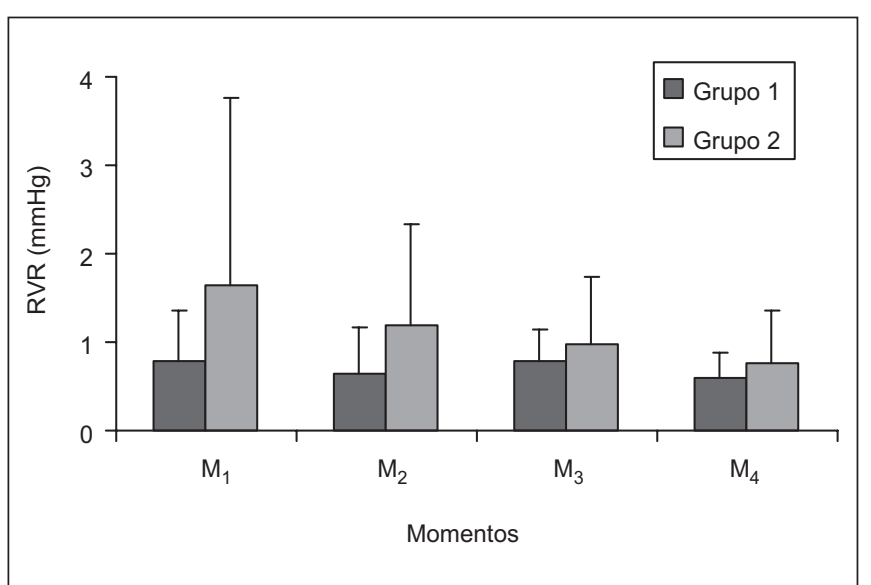

Figura 3 - Resistência Vascular Renal. Média e Desvio Padrão dos Valores Observados nos Dois Grupos Experimentais

Quanto aos íons sódio e potássio, os dois grupos apresentaram o mesmo comportamento, com os valores plasmáticos permanecendo estáveis durante o procedimento (Figuras 4 e 5).

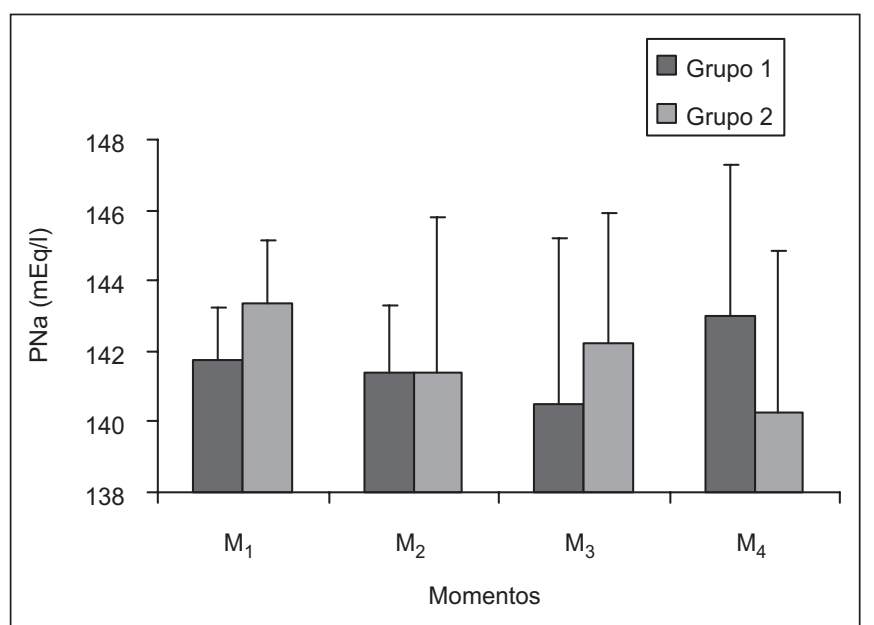

Figura 4 - Sódio Plasmático. Média e Desvio Padrão dos Valores Observados nos Dois Grupos Experimentais

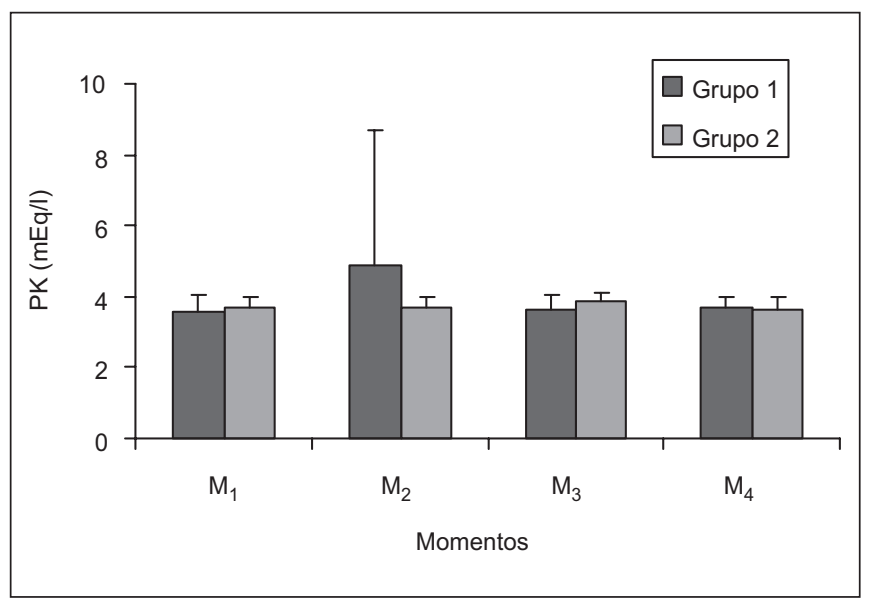

Figura 5 - Potássio Plasmático. Média e Desvio Padrão dos Valores Observados nos Dois Grupos Experimentais
Do mesmo modo, a osmolalidade plasmática e a depuração de creatinina apresentaram o mesmo comportamento em ambos os grupos, com os valores permanecendo estáveis durante o experimento (Figuras 6 e 7).

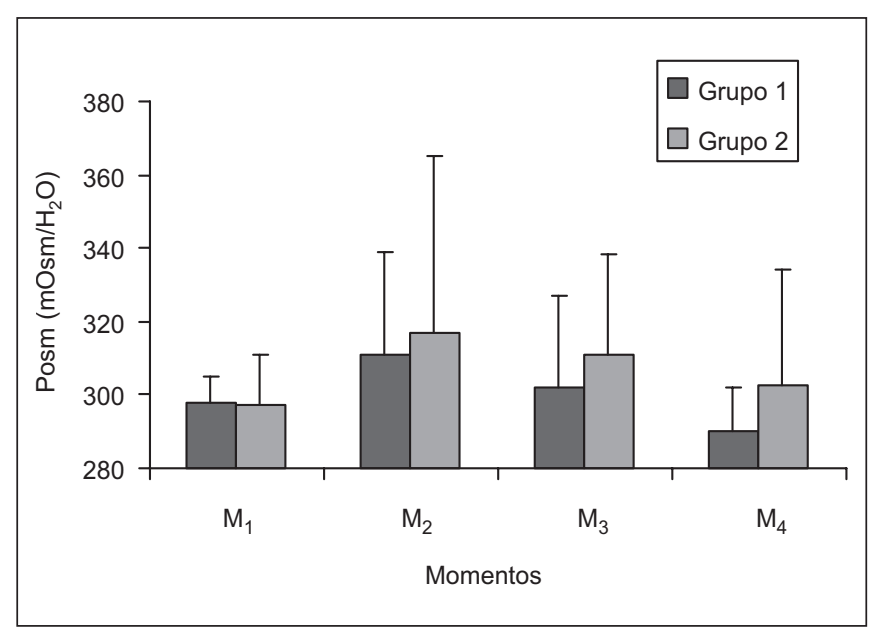

Figura 6 - Osmolaridade Plasmática. Média e Desvio Padrão dos Valores Observados nos Dois Grupos Experimentais

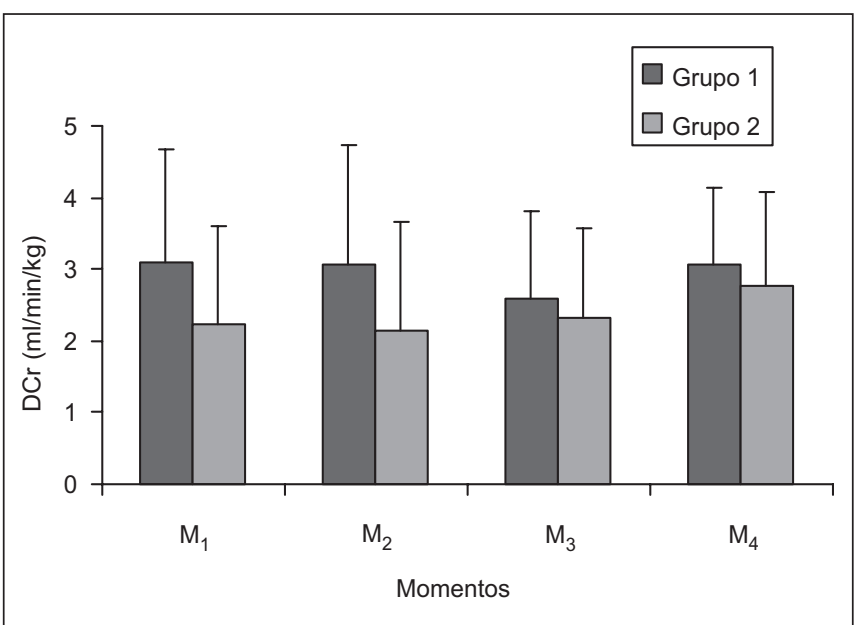

Figura 7 - Depuração de Creatinina. Média e Desvio Padrão dos Valores Observados nos Dois Grupos Experimentais

A fração de filtração também mostrou o mesmo comportamento em ambos os grupos, com redução significativa dos valores em $\mathrm{M}_{2}$, que assim permaneceram até o final do experimento (Figura 8).

O volume urinário dos animais permaneceu estável durante todo o experimento em ambos os grupos, com os animais do Grupo 1 apresentando valores significativamente maiores que os do Grupo 2 (Figura 9).

Revista Brasileira de Anestesiologia Vol. 54, No 3, Maio - Junho, 2004 


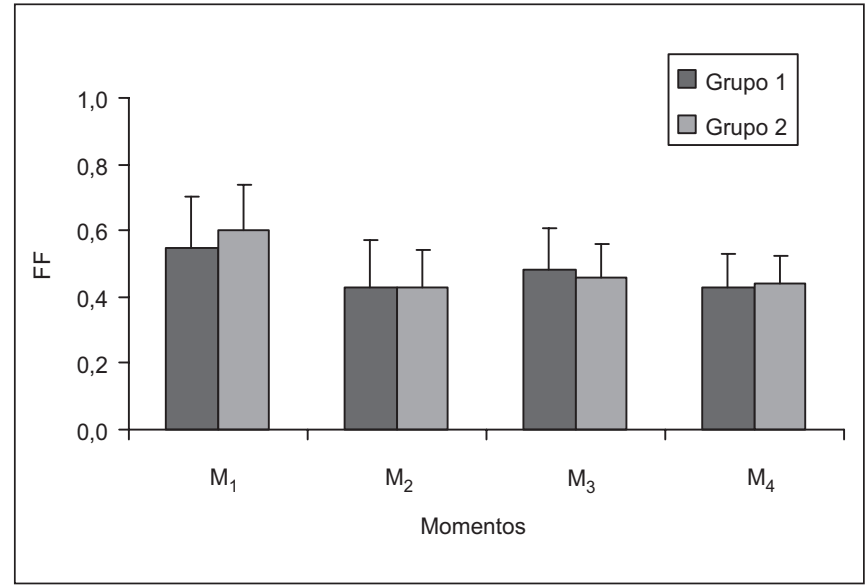

Figura 8 - Fração de Filtração. Média e Desvio Padrão dos Valores Observados nos Dois Grupos Experimentais

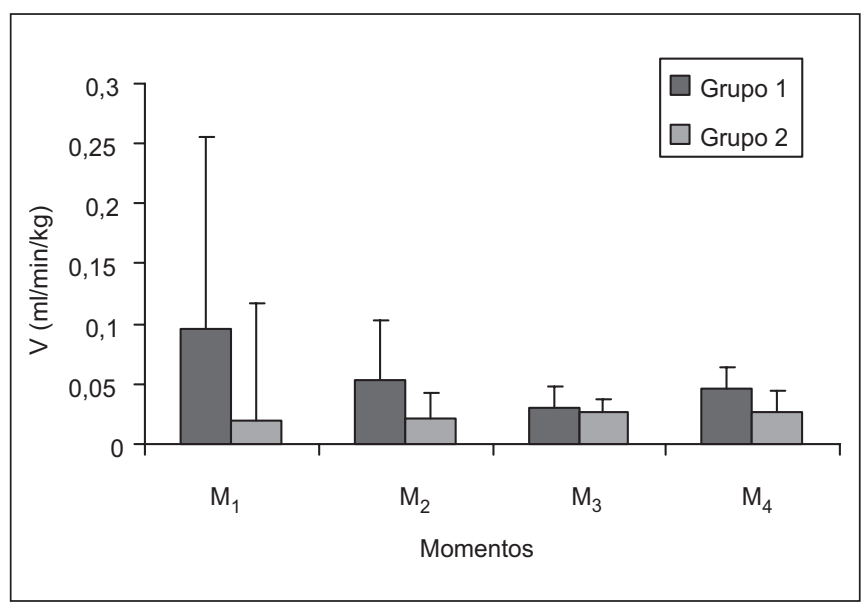

Figura 9 - Volume Urinário. Média e Desvio Padrão dos Valores Observados nos Dois Grupos Experimentais

Também a osmolalidade urinária de ambos os grupos apresentou o mesmo comportamento, com diminuição significativa no transcorrer do procedimento (Figura 10).

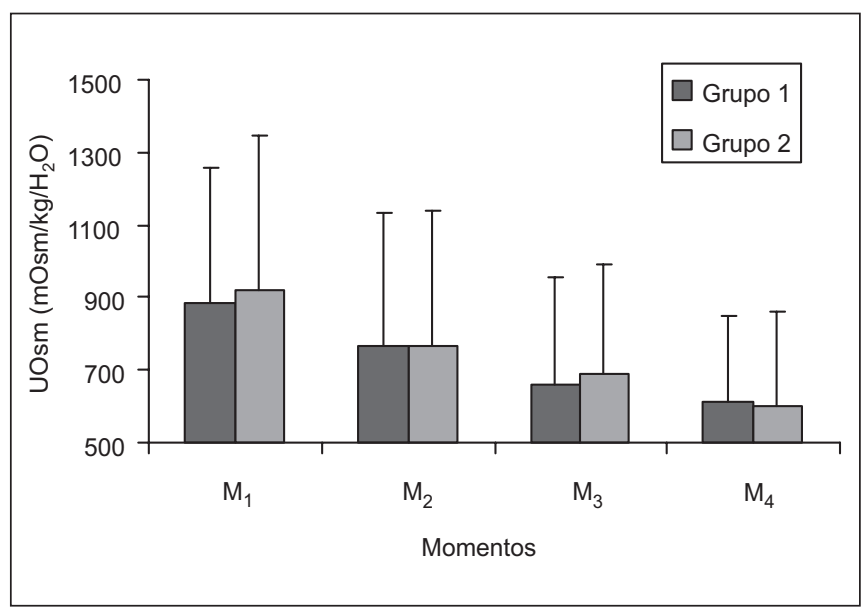

Figura 10 - Osmolalidade Urinária. Média e Desvio Padrão dos Valores Observados nos Dois Grupos Experimentais

Revista Brasileira de Anestesiologia

Vol. 54, № 3, Maio - Junho, 2004
Quanto à depuração osmolar, não houve variação entre os momentos nos dois grupos durante o experimento, apenas os valores do Grupo 1 sendo maiores que os do Grupo 2 (Figura 11).

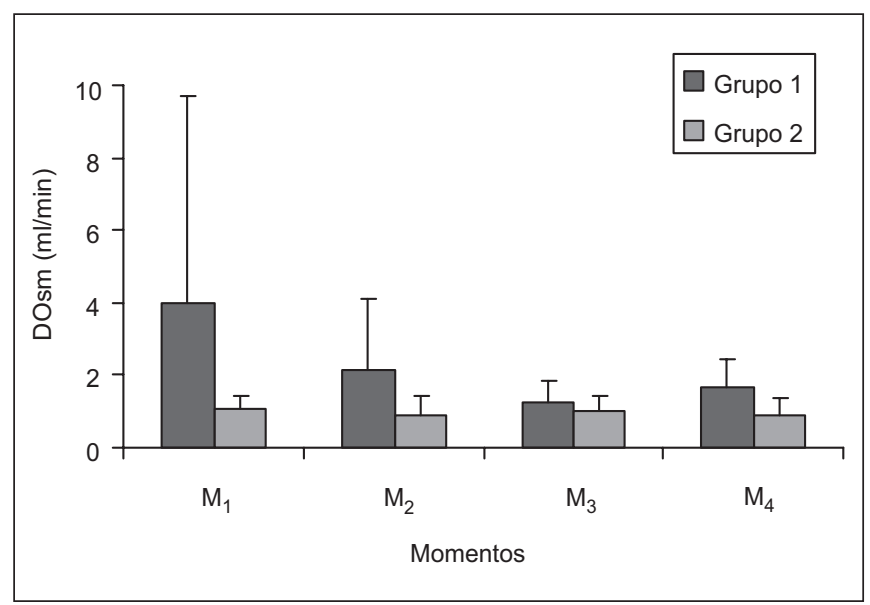

Figura 11 - Depuração Osmolar. Média e Desvio Padrão dos Valores Observados nos Dois Grupos Experimentais

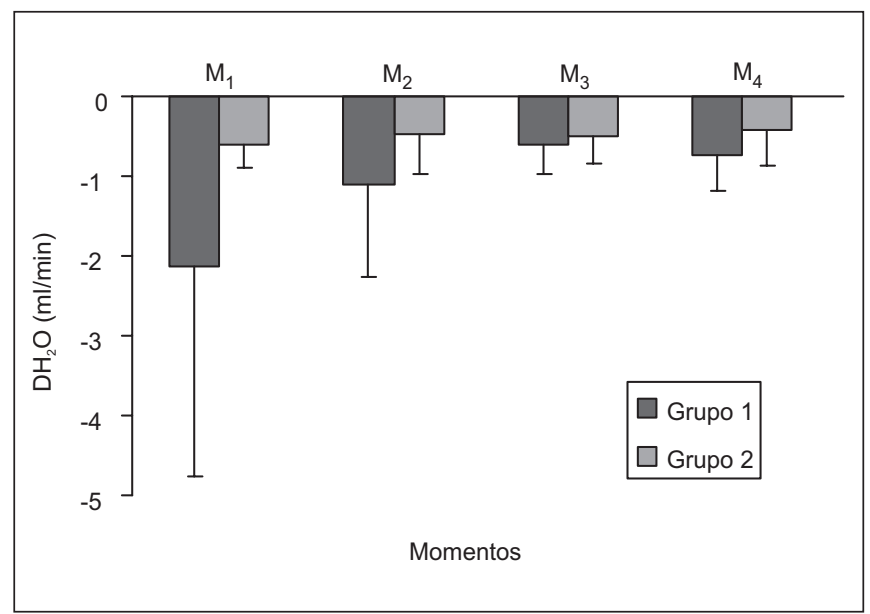

Figura 12 - Depuração de Água Livre. Média e Desvio Padrão dos Valores Observados nos Dois Grupos Experimentais

Já a depuração de água livre apresentou, no conjunto dos grupos, aumento em seus valores durante o experimento (Figura 12).

Quanto à depuração de sódio, excreção urinária de sódio e excreção fracionária de sódio, os grupos apresentaram perfis similares, não ocorrendo variação dos seus valores no transcorrer do experimento, apenas os valores do Grupo 1 sendo significativamente maiores que os do Grupo 2 (Figuras 13, 14 e 15). 


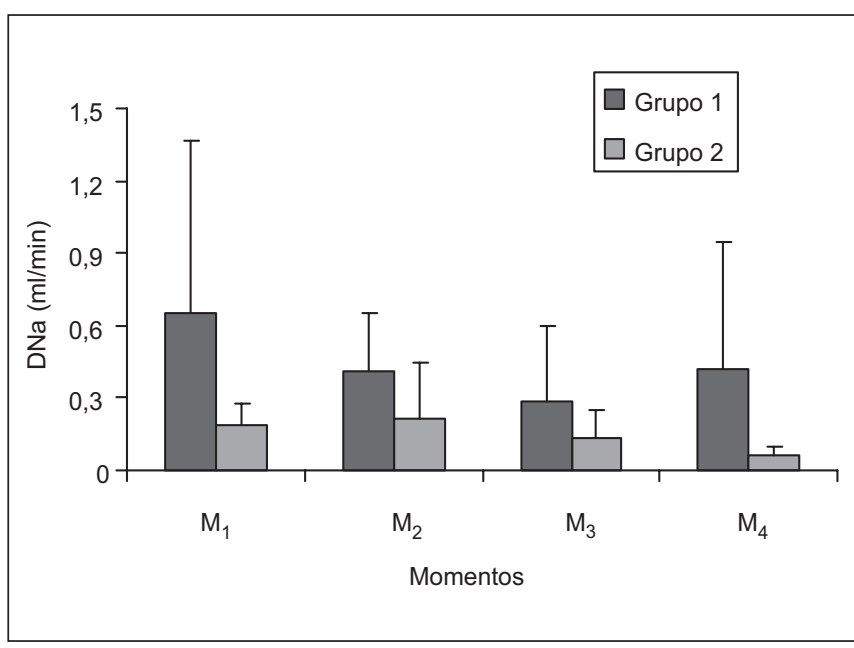

Figura 13 - Depuração de Sódio. Média e Desvio Padrão dos Valores Observados nos Dois Grupos Experimentais

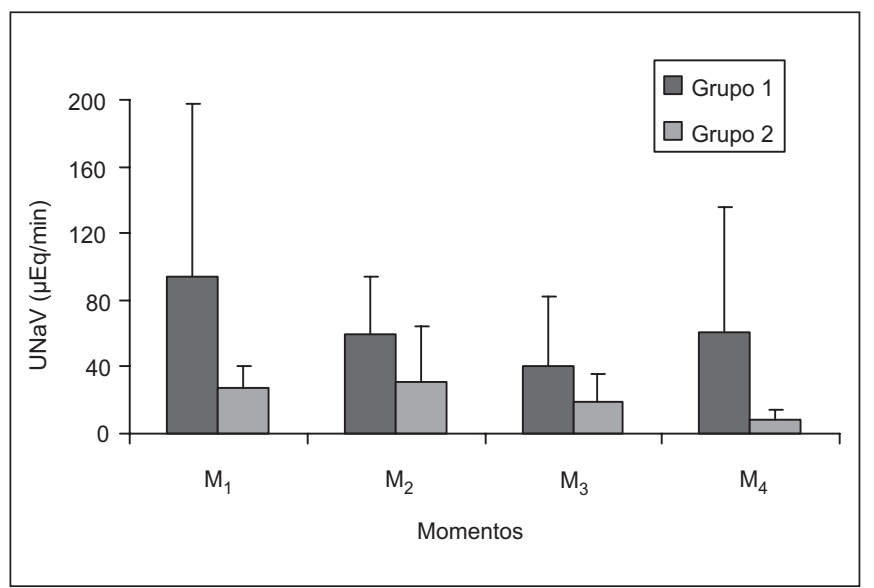

Figura 14 - Excreção Urinária de Sódio. Média e Desvio Padrão dos Valores Observados nos Dois Grupos Experimentais

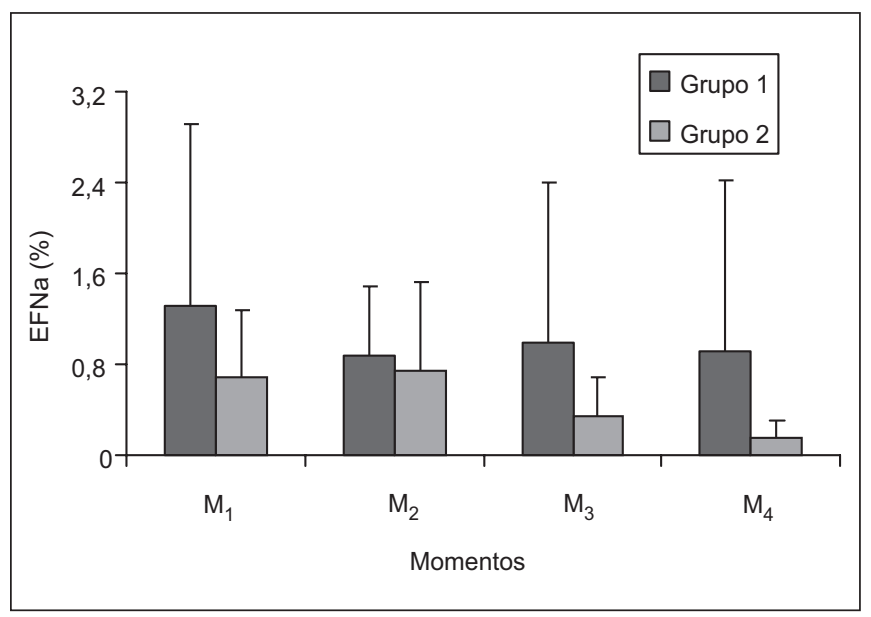

Figura 15 - Excreção Fracionária de Sódio. Média e Desvio Padrão dos Valores Observados nos Dois Grupos Experimentais
Quanto à depuração de potássio, G1 e G2 apresentaram comportamentos similares com diminuição significativa em $\mathrm{M}_{2}$, assim permanecendo até o final do experimento. Os valores do Grupo 1 foram significativamente superiores aos do Grupo 2 (Figura 16).

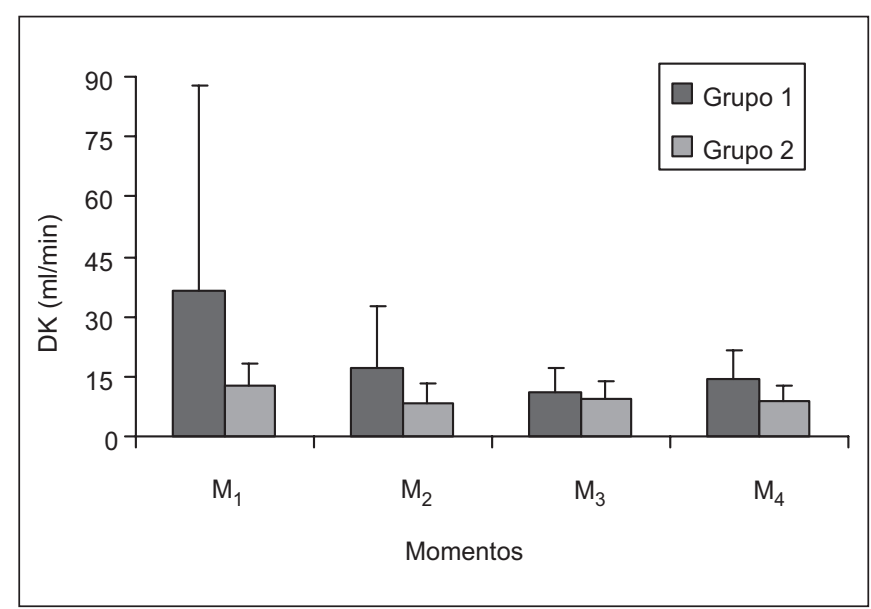

Figura 16 - Depuração de Potássio. Média e Desvio Padrão dos Valores Observados nos Dois Grupos Experimentais

Já a excreção urinária de potássio apresentou perfis similares, com valores permanecendo estáveis em ambos os grupos durante o experimento. Os valores do Grupo 1 foram significativamente superiores aos do Grupo 2 (Figura 17).

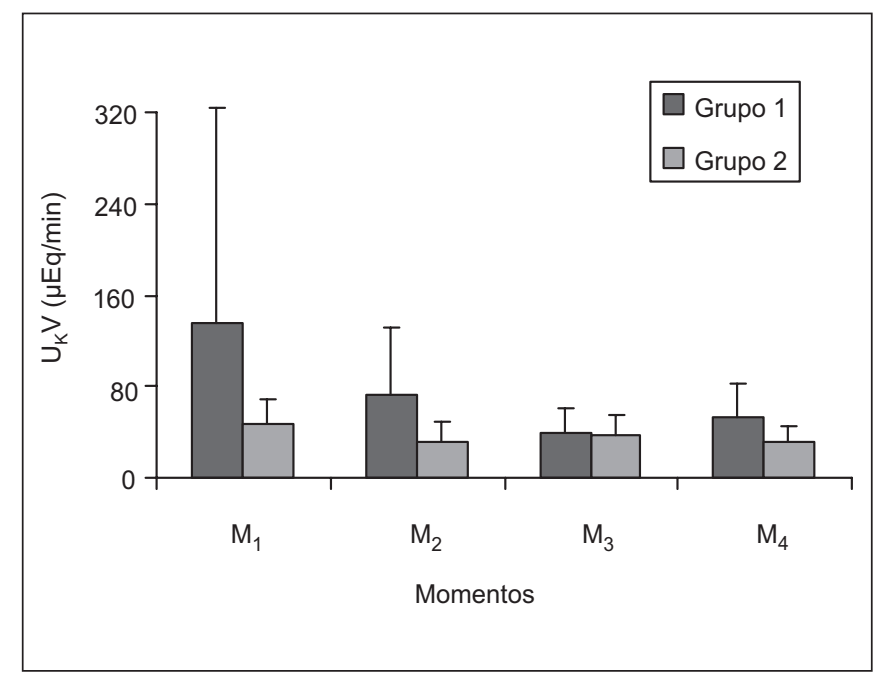

Figura 17- Excreção Urinária de Potássio. Média e Desvio Padrão dos Valores Observados nos Dois Grupos Experimentais

Os dois grupos apresentaram perfis similares e iguais com relação à excreção fracionária de potássio, que diminuiu em $\mathrm{M}_{2}$, permanecendo reduzida até o final do experimento (Figura 18). 


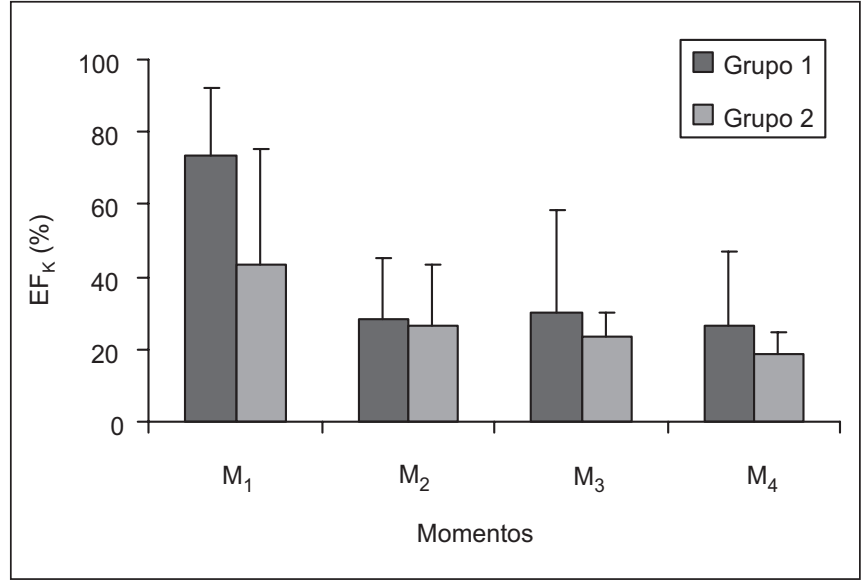

Figura 18 - Excreção Fracionária de Potássio. Média e Desvio Padrão dos Valores Observados nos Dois Grupos Experimentais

\section{DISCUSSÃO}

O aumento da pressão intra-abdominal (PIA) determinado pelo pneumoperitônio causa alterações sistêmicas, cuja magnitude está diretamente relacionada aos valores da PIA utilizados.

Em pacientes com comprometimento da função renal, seja ele agudo ou crônico, essas alterações podem ser importantes, uma vez que se observa freqüentemente diminuição da diurese durante o pneumoperitônio.

A compressão de artérias e veias, associada com compressão do parênquima renal e alterações hormonais, leva à diminuição da diurese ${ }^{14}$. Tal fato ocorre até mesmo em pacientes sem qualquer evidência de alteração histológica ou lesão tubular renal. Habitualmente não se observa comprometimento da diurese e função renal após a desinsuflação da cavidade peritoneal ${ }^{15}$.

Em estudo experimental, em cães, verificou-se diminuição de $63 \%$ do débito urinário e de $26 \%$ do fluxo sangüíneo renal, quando o rim estava sob efeito compressivo de PIA de 15 $\mathrm{mmHg}^{1}$. Já, quando a PIA foi superior a $20 \mathrm{mmHg}$, foi demonstrada redução de $75 \%$ da filtração glomerular, e anúria, com PIA de $40 \mathrm{mmHg}$, valores que não se modificaram com aumentos da hidratação ${ }^{2}$.

Em modelo utilizando porcos submetidos a diferentes valores de PIA durante 4 horas, verificou-se que pressões iguais ou maiores que $15 \mathrm{mmHg}$ provocaram diminuição do débito cardíaco, do débito urinário, do fluxo sangüíneo da veia renal e da depuração da creatinina. Estas alterações não foram associadas a lesões permanentes ou alterações histológicas renais ${ }^{16}$

Os estudos clínicos e experimentais sobre função renal e pneumoperitônio não apresentam uniformidade quanto ao volume de líquido que deve ser administrado, que varia de 2 a $15 \mathrm{ml} . \mathrm{kg}^{-1} \cdot \mathrm{h}^{-1}$. A oligúria parece não sofrer influência da hidratação $16-19$

Na presente pesquisa, os animais receberam hidratação de $6 \mathrm{ml} . \mathrm{kg}^{-1} \cdot \mathrm{h}^{-1} \mathrm{com}$ a finalidade de mantê-los hidratados, evitando-se a hiper-hidratação e a desidratação.
Em cirurgias laparoscópicas, os valores elevados de $\mathrm{CO}_{2}$ sangüíneo podem aumentar a atividade da renina plasmática, as concentrações plasmáticas de catecolaminas, de aldosterona e de vasopressina ${ }^{6,20,21}$. Como resultado desta estimulação hormonal, ocorre vasoconstrição renal com diminuição do fluxo sangüíneo renal e da filtração glomerular $^{22}$.

O rápido aumento da resistência vascular sistêmica que se desenvolve após a instalação do pneumoperitônio tem sido correlacionado a alterações hormonais, com aumento da atividade da renina plasmática ${ }^{6,7,9}$ e da vasopressina $3,18,23$

A utilização de um antagonista da vasopressina, SR 4925, inibiu o aumento da resistência vascular sistêmica ${ }^{23}$. Assim, a vasopressina possui papel importante na regulação hemodinâmica durante o pneumoperitônio ${ }^{23,24}$.

Já com relação às catecolaminas, não existe relação temporal entre o aumento de sua concentração plasmática e os aumentos da resistência vascular sistêmica e da pressão arterial média ${ }^{24}$.

Nesta pesquisa, os valores do $\mathrm{CO}_{2}$ sangüíneo mantiveram-se dentro dos limites fisiológicos, assim como as concentrações da vasopressina. A resistência vascular renal não variou no decorrer do experimento, em ambos os grupos, apenas os valores do Grupo 1 sendo maiores que os do Grupo 2, fato que relaciona-se à característica do grupo em estudo.

Em condições hemodinâmicas favoráveis, com o aumento do débito cardíaco e diminuição da resistência periférica, há aumento do fluxo sangüíneo renal, do fluxo plasmático renal e da fração de filtração; na realidade, observa-se diminuição da fração de filtração, isto é, da relação entre o ritmo de filtração glomerular e o fluxo plasmático renal, mostrando que o aumento do fluxo plasmático renal foi proporcionalmente maior que o do ritmo de filtração glomerular ${ }^{25}$.

Assim, a diminuição da fração de filtração observada neste estudo, no qual houve aumento do índice cardíaco e diminuição do índice de resistência vascular sistêmica ${ }^{26}$, seria decorrente de aumento do fluxo plasmático renal.

No fluido extracelular, o cátion sódio e os dois principais ânions que o acompanham, o cloro e o bicarbonato, são responsáveis por $90 \%$ da quantidade de solutos neste compartimento ${ }^{25}$. Assim sendo, pode-se estimar a osmolalidade plasmática pelas medidas da concentração de sódio plasmático.

A osmolalidade plasmática mantém-se praticamente sem variações devido à existência de integração hipotalâmica-hipofisária-renal e do hormônio antidiurético, o qual exerce função primordial na manutenção desta estabilidade ${ }^{27}$. O sódio plasmático não se alterou de maneira significativa em nenhum dos dois grupos de estudo durante o experimento, com estabilidade da osmolalidade plasmática e dos valores plasmáticos de vasopressina.

A ventilação com pressão positiva pode ocasionar diminuição do débito cardíaco e da pressão arterial em situações como hipovolemia e pressão intratorácica aumentada, como ocorre no pneumoperitônio ${ }^{28,29}$. 
As reduções do débito cardíaco e da pressão arterial causam diminuição do fluxo sangüíneo renal, ativação do sistema renina-angiotensina-aldosterona e maior reabsorção de sódio e água, alterando a osmolalidade plasmática.

Nesta pesquisa não foram observadas, em ambos os grupos, alterações no índice cardíaco, na pressão arterial média ${ }^{26}$ e no fluxo sangüíneo renal, não se esperando, portanto, influências na osmolalidade plasmática. Esta manteve-se estável no transcorrer do experimento; não tendo sofrido, portanto, influências do pneumoperitônio, da hidratação e da ventilação mecânica.

O evento mais marcante desencadeado pelo aumento da PIA sobre os rins é a diminuição da diurese ${ }^{1,8,17,19,30}$. Ela está relacionada ao aumento da concentração plasmática da vasopressina ${ }^{3,8,23}$ e também à compressão mecânica sobre o parênquima renal, da artéria renal, da veia renal e da veia cava inferior, que desencadeia efeitos negativos sobre o fluxo sangüíneo renal e a função renal.

A diurese está aumentada quando há expansão do volume extracelular ${ }^{25}$, liberação do hormônio natriurético ${ }^{31}$, estímulo dos receptores de estiramento e de pressão atrial ${ }^{32} \mathrm{e}$ aumento da pré-carga.

A ventilação pulmonar mecânica, por sua vez, porque determina aumentos na pressão intratorácica e, conseqüentemente, redução do retorno venoso e do débito cardíaco, pode estimular a liberação de vasopressina e a inibição do fator natriurético atrial. No presente estudo, entretanto, não foram observadas diminuições no índice cardíaco ${ }^{26}$ ou alterações nos valores de vasopressina plasmática em ambos os grupos.

Nesta pesquisa, a vasopressina, que desempenha papel importante na perfusão renal e na reabsorção de água, não se alterou e a PIA não excedeu $15 \mathrm{mmHg}$ durante uma hora. Estes fatores, associados ao padrão de hidratação estabelecido, com manutenção de normovolemia, podem ter contribuído para a manutenção da diurese durante todo o procedimento em ambos os grupos. Os maiores valores observados no Grupo 1 seriam características próprias e condizentes com os maiores valores de pressão arterial média apresentados pelo mesmo.

Considerando-se que houve manutenção da normovolemia, estabilidade hemodinâmica e ausência de variação nos valores da vasopressina plasmática em ambos os grupos, pode-se inferir que a ausência de variações significativas no sódio plasmático, na depuração de sódio, na excreção urinária de sódio e na excreção fracionária de sódio foi reflexo da estabilidade hemodinâmica determinada pelas modalidades ventilatórias.

É conhecido que a polaridade estrutural das células do túbulo proximal é essencial para a função primária da reabsorção seletiva de moléculas do fluido tubular e que alterações nesta ocorrem durante isquemia renal ${ }^{33}$. Mudanças na posição da bomba $\mathrm{Na}$, K-ATPase são responsáveis pela alta fração de excreção de sódio, que é característica da urina de pacientes com falência renal aguda pós-isquêmica ${ }^{34}$.

Há que ressaltar que não existiu, em nenhum momento, em ambos os grupos aumento na excreção renal de sódio, mas, ao analisar-se os valores absolutos da depuração de sódio, da excreção urinária de sódio e da excreção fracionária de sódio, pode-se inferir que ocorreu tendência à diminuição dos seus valores, em ambos os grupos, no transcorrer do experimento, o que vem se somar à diminuição da osmolalidade urinária e ao aumento da depuração de água livre.

Assim, tendências à diminuição na depuração e na excreção urinária e fracionária de sódio poderiam revelar discreto grau de comprometimento da função renal desencadeado pelo aumento da PIA, levando à compressão do parênquima e determinando possíveis alterações de absorção e secreção tubulares.

Os maiores valores desses atributos observados em G1 podem ser reflexos dos maiores valores na pressão arterial média observados em 1 $^{25,26}$

Quanto ao potássio, acredita-se que quase tudo o que é filtrado seja reabsorvido e que o potássio excretado na urina tenha origem na secreção tubular. Quando o fluido tubular atinge o túbulo distal, $85 \%$ a $95 \%$ do potássio filtrado já foram reabsorvidos, as concentrações e quantidades excretadas na urina final não apresenta grandes variações ${ }^{35}$.

A regulação da excreção do potássio sofre influência das suas concentrações plasmática e celular, as quais, quando aumentadas, também atuam na secreção adrenal de aldosterona, que estimula a reabsorção de sódio e a excreção de potássio, não existindo necessariamente um acoplamento obrigatório entre estes mecanismos celulares de absorção e excreção ${ }^{36}$.

A secreção do potássio no túbulo distal pode estar aumentada em função de sobrecarga de fluidos e por aumento do fluxo luminal ${ }^{36}$. A expansão volêmica tem a propriedade de diminuir a atividade da renina plasmática com conseqüente redução da formação de aldosterona e menor excreção de potássio. O fluxo intratubular aumentado diminui a concentração luminal de potássio, favorecendo movimento iônico passivo da célula para a luz tubular, na direção do gradiente do íon.

No equilíbrio ácido-base existe correlação inversa entre os valores de excreção de ácido e secreção de potássio. Estados alcalóticos levam à maior excreção de potássio; ao contrário, a excreção de potássio diminui nos estados acidóticos ${ }^{36}$. A relação entre o $\mathrm{pH}$ plasmático e o potássioé tal que, para cada aumento ou diminuição de 0,1 unidade de $\mathrm{pH}$, ocorre diminuição ou elevação de $0,63 \mathrm{mEq} / \mathrm{l}$ de potássio no plasma.

As modalidades ventilatórias utilizadas determinaram diminuição nos valores do $\mathrm{pH}$, mais evidente no Grupo 1, o que poderia culminar com menor depuração e excreção fracionária de potássio.

A ventilação com pressão positiva pode atuar sobre o potássio por meio da ação hormonal da aldosterona, induzindo a maior reabsorção de sódio e excreção de potássio. Tal fato, entretanto, não ocorreu uma vez que a excreção fracionária de potássio não aumentou, mostrando que as modalidades ventilatórias não interferiram com o sistema renina-angiotensina-aldosterona. Vol. 54, No 3, Maio - Junho, 2004 
Em estudo clínico desenvolvido com pacientes submetidos à colecistectomia laparoscópica, não se observou alterações na excreção fracionária de potássio ${ }^{37}$. Já em porcos submetidos a pneumoperitônio, foi constatado aumento nas concentrações plasmáticas de potássio ${ }^{16,38}$. Em ambos os estudos as concentrações séricas alcançadas ficaram abaixo de valores potencialmente capazes de desencadear disritmias cardíacas.

Neste experimento, as concentrações do potássio sérico não se alteraram. Contudo, a depuração e a excreção fracionária de potássio diminuíram a partir da obtenção do pneumoperitônio, e a excreção urinária de potássio apresentou tendência à diminuição em ambos os grupos. A maior depuração e excreção fracionária de potássio do Grupo 1 provavelmente decorreu do maior valor de pressão arterial média apresentado por este grupo.

É provável que não tenha ocorrido influência da aldosterona no manuseio do potássio, visto que não foi observado aumento na reabsorção de sódio, o que levaria à excreção do íon. Como os parâmetros hemodinâmicos permaneceram estáveis, o estado volêmico do animal foi suficiente para não influenciar o fluxo tubular.

Apesar da concentração plasmática de potássio não ter se alterado, alguns fatores como a ação compressiva sobre o parênquima renal determinada pelo aumento da PIA e/ou a diminuição do $\mathrm{pH}$ arterial poderiam contribuir para as menores depuração e excreção fracionária do íon observadas em ambos os grupos a partir de $\mathrm{M}_{2}$, decorrentes de sua maior reabsorção e/ou menor secreção.

O transporte da água é sempre secundário ao transporte de outros solutos, realizado por mecanismos puramente físico-químicos. Nos segmentos mais distais do néfron, o transporte de água é modulado pelo hormônio antidiurético ou vasopressina $^{39}$.

A relação entre a excreção de solutos e a água pode ser estudada considerando-se que o volume urinário seja constituído por "duas águas" - uma para conter todos os solutos (osmoles) eliminados na urina e outra "água" a ser eliminada como tal, pura, livre de solutos ${ }^{40}$.

A diminuição da osmolalidade urinária observada após a instalação do pneumoperitônio pode ter sido conseqüência da menor depuração e excreção do íon potássio, somada à tendência de menor depuração e excreção do íon sódio.

A maior depuração de água livre, observada a partir da instalação do pneumoperitônio, também está em concordância com a diminuição da osmolalidade urinária, representando a tendência de filtrado urinário maior e livre de solutos. Os valores plasmáticos de vasopressina mantiveram-se dentro da normalidade, o que exclui a participação do hormônio no aumento da depuração de água livre.

Adepuração osmolar, que corresponde ao volume de plasma (ml) que se liberta de todas as substâncias osmoticamente ativas na unidade de tempo, não variou estatisticamente em ambos os grupos.

A manutenção dos valores da depuração do $\mathrm{PAH}$ durante todo o procedimento reflete um ritmo de filtração glomerular estável, excluindo-se, desta forma, qualquer comprometimento da função renal secundária a fatores pré-renais.

Assim, a diminuição da osmolalidade urinária e o aumento na depuração de água livre poderiam ser decorrentes do comprometimento do parênquima renal, como conseqüência do aumento da PIA, que exerceria efeito compressivo sobre o rim, levando a alterações na capacidade de absorção e secreção dos néfrons.

Portanto, pode-se concluir que, dentre as inúmeras variáveis que poderiam determinar alterações na hemodinâmica e função renais, neste experimento, as modalidades ventilatórias não levaram a alterações sistêmicas capazes de determinar comprometimento da função renal. Pode-se inferir que a compressão do parênquima renal, determinada pelo aumento da pressão intra-abdominal, pode ter sido responsável por alterações na excreção renal do potássio.

\section{Effects of Pneumoperitoneum on Renal Hemodynamics and Function of Dogs under Volume and Pressure-Controlled Ventilation}

Armando Vieira de Almeida, TSA, M.D; Eliana Marisa Ganem, TSA, M.D.

\section{INTRODUCTION}

Increased abdominal pressure promoted by pneumoperitoneum as well as intraoperative patients' positioning may affect several organs and systems during videolaparoscopic surgeries.

Compression of renal parenchyma, arteries and veins during pneumoperitoneum peaks with oliguria ${ }^{1}$. When intra-abdominal pressure increases from 0 to $20 \mathrm{mmHg}$, renal vascular resistance increases $555 \%$ and glomerular filtration rate decreases $25 \%^{2}$.

Renal changes are also associated to neuroendocrine system activation ${ }^{3,4}$. High plasma vasopressin ${ }^{3-5}$, rennin ${ }^{4,6-9}$ and endothelin ${ }^{10}$ concentrations have been observed during pneumoperitoneum. The increase of these substances in plasma induces vasoconstriction and contributes for systemic vascular resistance increase.

Rennin-angiotensin-aldosterone system activation leads to angiotensin II-mediated renal vasoconstriction promoting medulla blood flow shift to renal cortex, worsening renal perfusion ${ }^{8}$.

When mechanical ventilation is installed, internal chest pressure variations change pre and afterload, which may affect cardiovascular hemodynamics ${ }^{11}$ and renal hemodynamics and function, especially in severely ill patients. Added to this, there is IAP increase which, depending on clinical conditions and patients' positioning, may determine major changes in 
established ventilatory parameters which, as a consequence, may lead to hemodynamic changes.

Oliguria is a common event during pneumoperitoneum, evidencing not yet explained renal homeostasis changes. This study aimed at correlating two ventilatory modes - volume and pressure controlled - to renal hemodynamics and function changes in dogs submitted to 10 and $15 \mathrm{mmHg}$ pneumoperitoneum.

\section{METHODS}

After the Animal Experiment Ethics Committee approval, 16 adult mixed-breed dogs of both genders, weighing 15 to $23 \mathrm{~kg}$ and supplied by the Experimental Animals Facility, Botucatu Campus, Universidade Estadual Paulista Julio de Mesquita Filho were evaluated.

Exclusion criteria were unhealthy-looking animals. Dogs were randomly distributed in two experimental groups of 8 animals according to the ventilatory mode:

Group 1 (G1) - Volume controlled ventilation;

Group 2 (G2) - Pressure controlled ventilation.

After 12-hour fasting with free access to water, animals were weighed, anesthetized with intravenous $15 \mathrm{mg} \cdot \mathrm{kg}^{-1}$ sodium pentobarbital and $15 \mu \mathrm{g} . \mathrm{kg}^{-1}$ fentanyl and placed in the supine position on Claude Bernard's device, when they were ventrally measured from nose to anus. Using this distance and weight in $\mathrm{kg}$, body surface was estimated through normal physiological data tables.

Then, animals were intubated with 38 tube with low pressure and high compliance cuff to install mechanical ventilation. Animals were ventilated with $100 \%$ oxygen. Group 1, with volume controlled ventilation by Ohmeda's anesthesia machine and monitored by Datex-Engstrom's AS/3 device, received tidal volume enough to maintain $\mathrm{P}_{\mathrm{ET}} \mathrm{CO}_{2}$ (end tidal $\mathrm{CO}_{2}$ ) between 35 and $45 \mathrm{mmHg}$. Group 2, with pressure controlled ventilation by Ohmeda's anesthesia machine and monitored by Datex-Engstrom's AS/3 device, was ventilated with enough pressure to maintain $\mathrm{P}_{E T} \mathrm{CO}_{2}$ between 35 and 45 $\mathrm{mmHg} . \mathrm{P}_{\mathrm{ET}} \mathrm{CO}_{2}$ was measured by expired air sample collection close to the $Y$ piece of the respiratory circuit. Respiratory rate was initially established in 10 movements per minute for both groups. To help artificial ventilation, pancuronium was administered in the initial dose of $0.07 \mathrm{mg} . \mathrm{kg}^{-1}$ and additional dose of $0.008 \mathrm{mg} \cdot \mathrm{kg}^{-1}$.

Right and left femoral veins and right and left femoral arteries were dissected and catheterized with polyethylene catheters introduced 2 to $3 \mathrm{~cm}$. Left femoral vein catheter received 6 $\mathrm{mL} . \mathrm{kg}^{-1} \cdot \mathrm{h}^{-1}$ lactated Ringer's solution controlled by Abbott Laboratories (USA) "Anne" ${ }^{\circledR}$ microprocessed pump, and sodium thiopental $\left(100 \mu \mathrm{g} \cdot \mathrm{kg}^{-1} \cdot \mathrm{min}^{-1}\right)$ and fentanyl $(0.1$ $\left.\mu \mathrm{g} \cdot \mathrm{kg}^{-1} \cdot \mathrm{min}^{-1}\right)$ continuous infusion.

Intravenous creatinine $\left(30 \mathrm{mg}^{-\mathrm{kg}^{-1}}\right)$ and sodium para-aminohippurate $\left(4 \mathrm{mg} . \mathrm{kg}^{-1}\right)$ prime was administered 30 minutes after lactated Ringer's solution infusion. Then, creatinine $(0.6 \mathrm{~g} \%)$ and $\mathrm{PAH}(0.24 \mathrm{~g} \%)$ continuous infusion in lactated Ringer's was installed until experiment completion, being administered $0.6 \mathrm{mg}$ creatinine and $0.24 \mathrm{mg} \mathrm{PAH} \mathrm{per}$ minute/kg, with infusion controlled by "Anne" microprocessed pump.

Left femoral artery catheter edge was connected to blood pressure reading module of the Datex-Engstrom's AS/3 device. Right femoral artery catheter was used to collect arterial blood.

After right cervical region tricotomy, right external jugular vein was dissected for Swan-Ganz catheter insertion according to Gouvea et al. ${ }^{12}$ technique.

Peritoneal cavity was accessed with the help of approximately 5 $\mathrm{cm}$ surgical incision on medial abdominal wall region, through which a $12 \mathrm{G}$ polyethylene catheter was inserted under direct view followed by surgical plane suture. Catheter edge was connected to the inflating device and pneumoperitoneum was achieved with $\mathrm{CO}_{2}$. Intra-abdominal $10 \mathrm{mmHg}$ and $15 \mathrm{mmHg}$ pressures were reached and maintained by the pressure and flow control module. Peritoneal cavity was deflated after disconnecting the catheter from the inflating device, followed by mild abdominal cavity compression.

Uretral catheterization was achieved with polyvinyl tube and vesical emptying was obtained with mild suprapubic compression.

For clearance periods (30 minutes), urine was collected in graded pipettes. In the middle of the procedure, $5 \mathrm{~mL}$ venous blood were collected in centrifuge tubes with heparin.

The following parameters: renal blood flow, renal vascular resistance, PAH clearance, plasma sodium, plasma potassium, plasma osmolality, creatinine clearance, filtration fraction, urinary volume, urinary osmolality, osmolar clearance, free water clearance, sodium clearance, sodium urinary excretion, sodium fractional excretion, potassium clearance, potassium urinary excretion and potassium fractional excretion were obtained in the following moments after $\mathrm{PAH}$ and creatinine prime injection:

$\mathrm{M}_{1}-60$ minutes after $\mathrm{PAH}$ and creatinine prime injection and immediately before $10 \mathrm{mmHg}$ pneumoperitoneum;

$\mathrm{M}_{2}-90$ minutes after $\mathrm{PAH}$ and creatinine prime injection, 30 minutes after $10 \mathrm{mmHg}$ pneumoperitoneum and immediately before $15 \mathrm{mmHg}$ pneumoperitoneum;

$\mathrm{M}_{3}-120$ minutes after $\mathrm{PAH}$ and creatinine prime injection, 30 minutes after $15 \mathrm{mmHg}$ pneumoperitoneum and immediately before pneumoperitoneum deflation;

$\mathrm{M}_{4}$ - 150 minutes after PAH and creatinine prime injection, 30 minutes after pneumoperitoneum deflation and immediately before animals' sacrifice.

For vasopressin dosage, $5 \mathrm{~mL}$ venous blood were collected in refrigerated and heparin-containing centrifuge tubes, in the following moments:

$\mathrm{M}_{1}{ }^{\prime}$ - immediately before $10 \mathrm{mmHg}$ pneumoperitoneum;

$\mathrm{M}_{2}{ }^{\prime}$ - 15 minutes after $10 \mathrm{mmHg}$ pneumoperitoneum;

$\mathrm{M}_{3}{ }^{\prime}$ - 15 minutes after $15 \mathrm{mmHg}$ pneumoperitoneum;

$\mathrm{M}_{4}{ }^{\prime}$ - 15 minutes after pneumoperitoneum deflation. 
Vasopressin was dosed by the radioimmunoassay technique using Arginine Vasopressin Radioimmunoassay DSL-1800 Kit.

At experiment completion animals were sacrificed with $10 \mathrm{~mL}$ intravenous injection of $19.1 \%$ potassium chloride.

For statistical analysis, Profile Analysis was used for Morrison's hypothesis test ${ }^{13}$. In all tested hypotheses, calculated F statistics were considered significant when $p<0.05$.

\section{RESULTS}

Statistical analysis of PAH clearance (Figure 1) and renal blood flow (Figure 2) has shown no differences between groups among moments throughout the experiment.

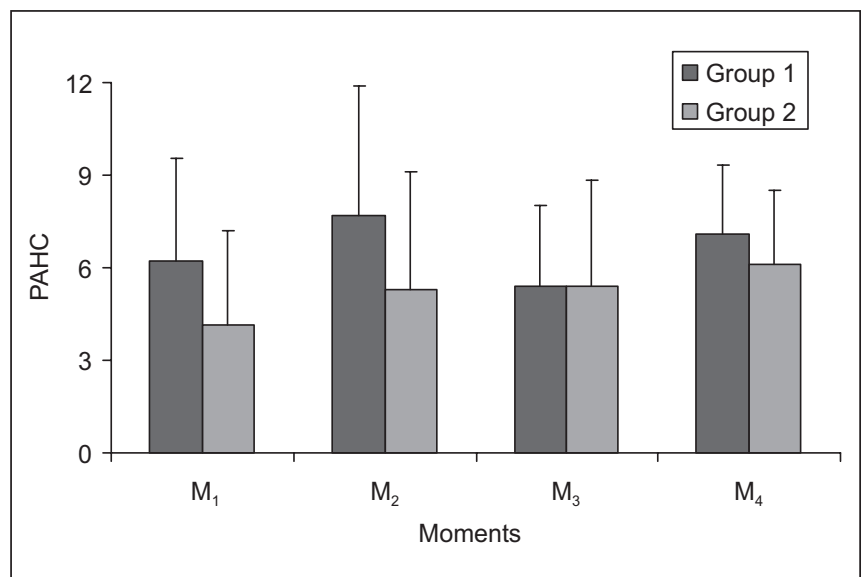

Figure 1 - Sodium Para-Aminohippurate Clearance. Mean and Standard Deviation in Both Experimental Groups

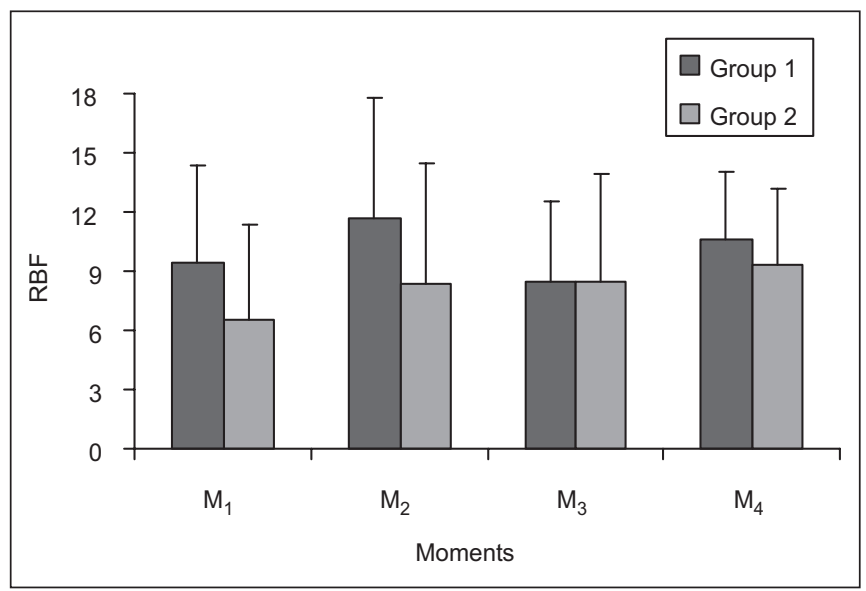

Figure 2 - Renal Blood Flow. Mean and Standard Deviation in Both Experimental Groups

Statistical analysis of renal vascular resistance has shown that it has been significantly lower in Group 1, however without differences among moments throughout the experiment for both groups (Figure 3).

Revista Brasileira de Anestesiologia

Vol. 54, № 3, Maio - Junho, 2004

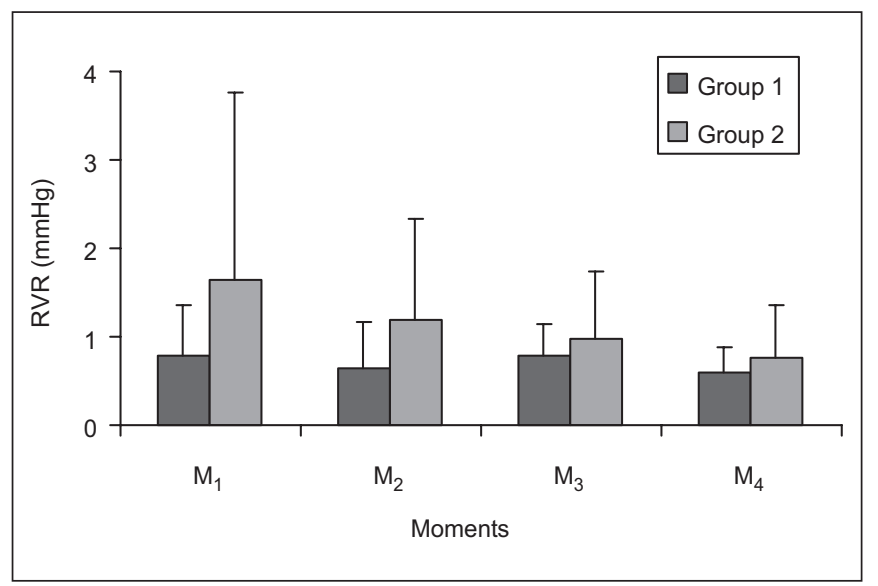

Figure 3 - Renal Vascular Resistance. Mean and Standard Deviation in Both Experimental Groups

Plasma sodium and potassium were similar for both groups with stable values throughout the procedure (Figures 4 and 5).

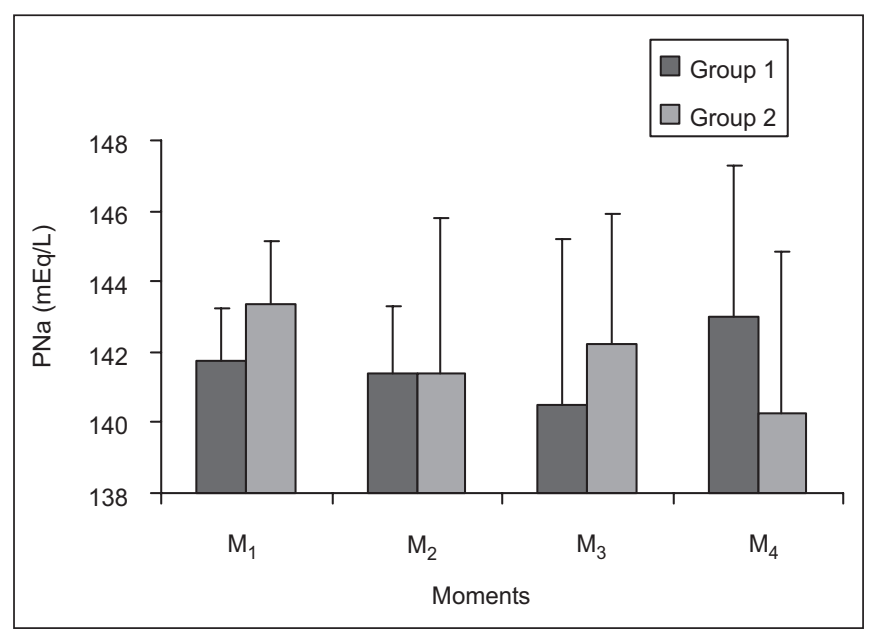

Figure 4 - Plasma Sodium. Mean and Standard Deviation in Both Experimental Groups

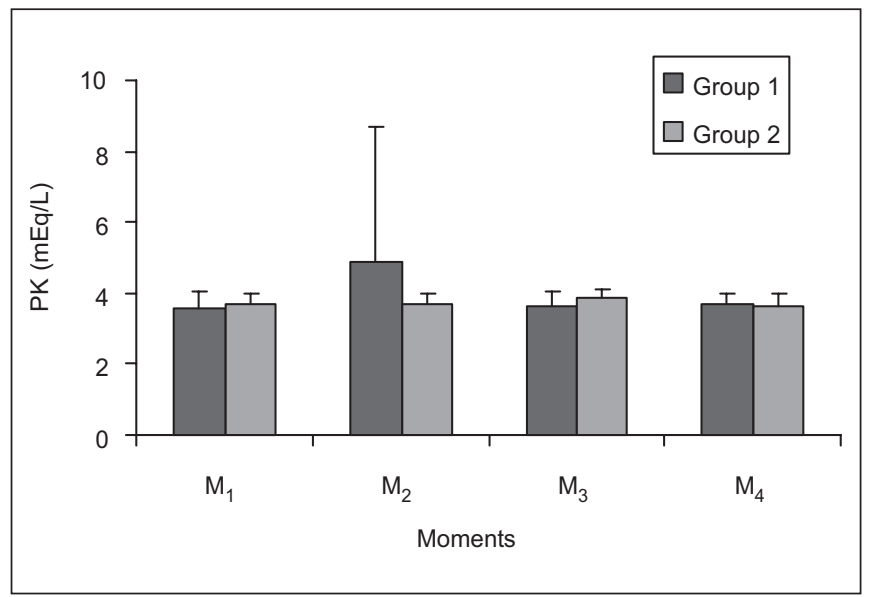

Figure 5 - Plasma Potassium. Mean and Standard Deviation in Both Experimental Groups 
Similarly, plasma osmolality and creatinine clearance were similar for both groups with stable values throughout the procedure (Figures 6 and 7).

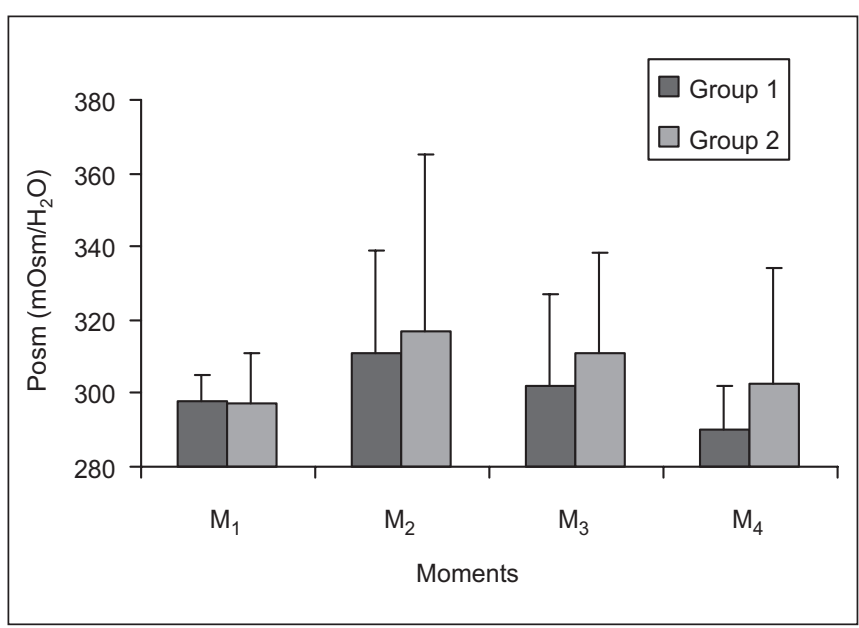

Figure 6 - Plasma Osmolality. Mean and Standard Deviation in Both Experimental Groups

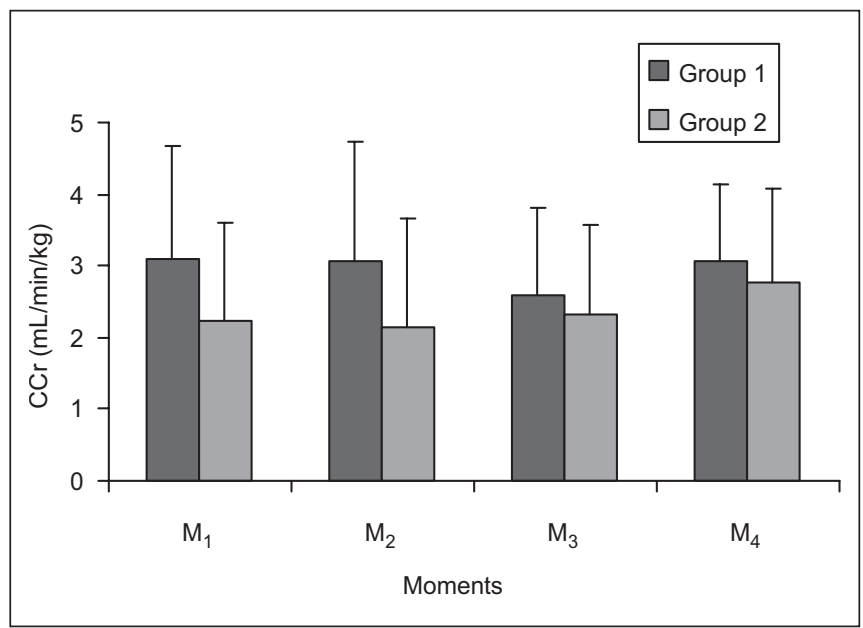

Figure 7 - Creatinine Clearance. Mean and Standard Deviation in Both Experimental Groups

Filtration fraction was also similar for both groups with significant decrease in $\mathrm{M}_{2}$ and remaining like that until experiment completion (Figure 8).

Urinary volume has been stable throughout the experiment for both groups, but Group1 had significantly higher values as compared to Group 2 (Figure 9).

Urinary osmolality was also similar for both groups with significant decrease throughout the procedure (Figure 10).

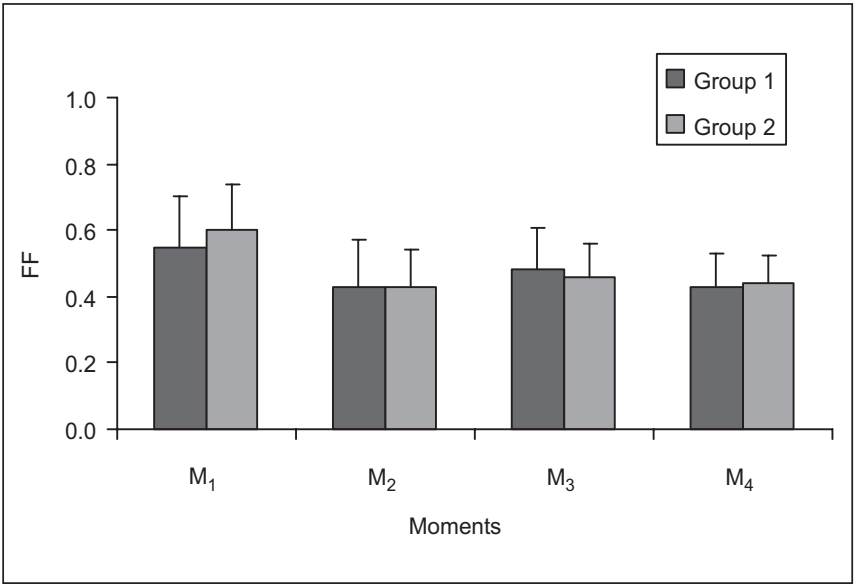

Figure 8 - Filtration Fraction. Mean and Standard Deviation in Both Experimental Groups

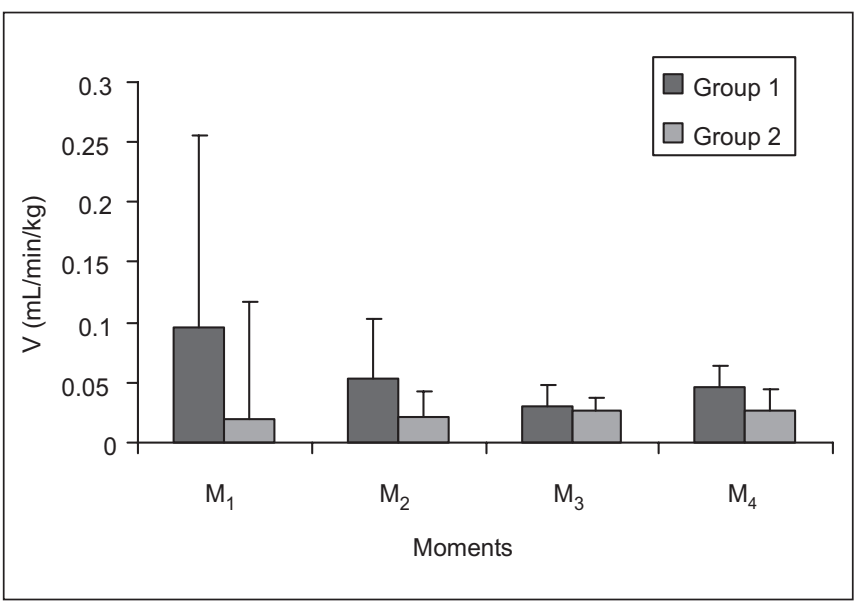

Figure 9 - Urinary Volume. Mean and Standard Deviation in Both Experimental Groups

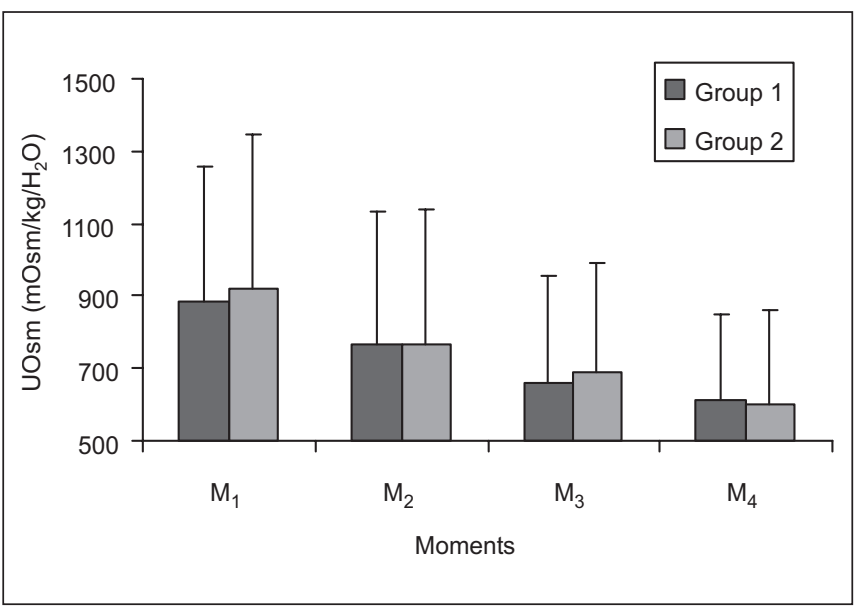

Figure 10 - Urinary Osmolality. Mean and Standard Deviation in Both Experimental Groups 
There has been no variation in osmolar clearance among moments in both groups, however Group 1 had higher values as compared to Group 2 (Figure 11).

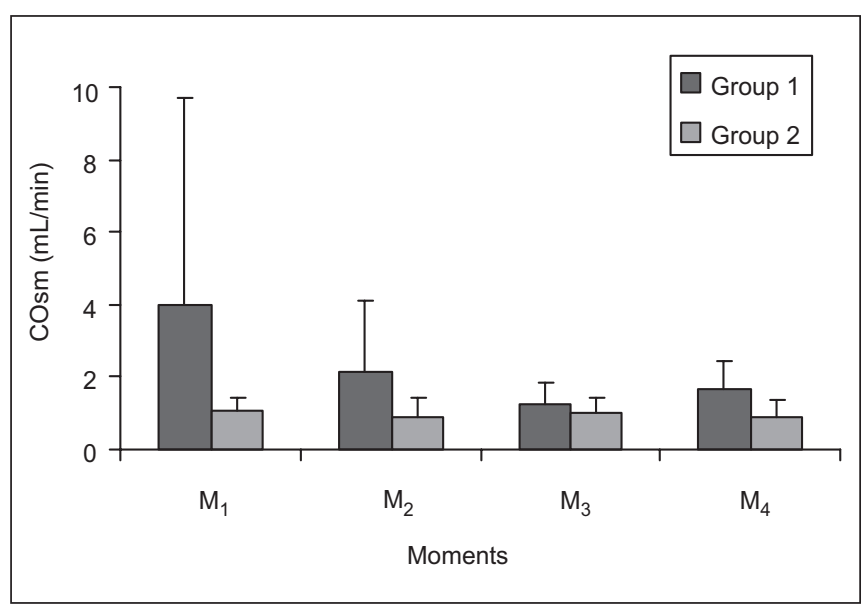

Figure 11 - Osmolar Clearance. Mean and Standard Deviation in Both Experimental Groups

Free water clearance has increased throughout the experiment for both groups (Figure 12).

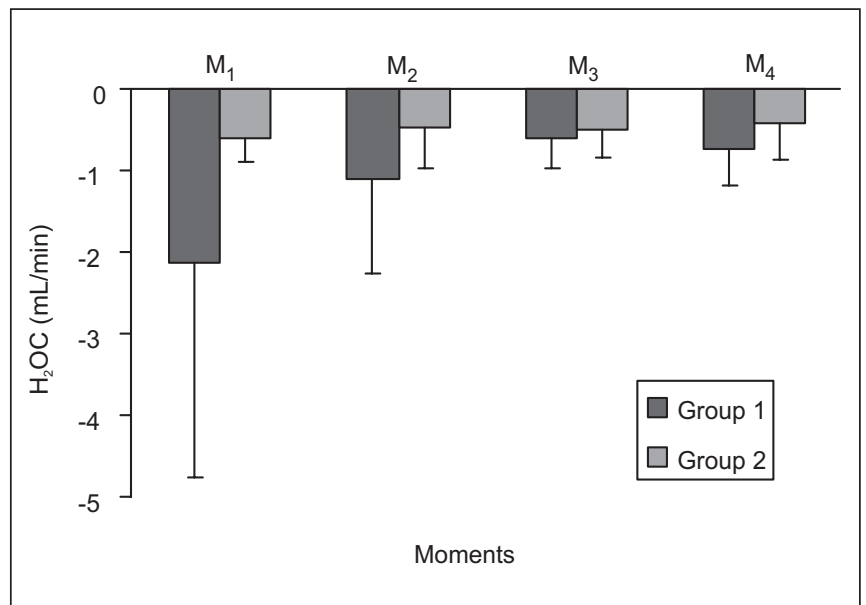

Figure 12 - Free Water Clearance. Mean and Standard Deviation in Both Experimental Groups

Sodium clearance, sodium urinary excretion and sodium fractional excretion were similar for both groups without variations throughout the experiment. Group 1, however, had significantly higher values as compared to Group 2 (Figures 13, 14 and 15).

Revista Brasileira de Anestesiologia Vol. 54, № 3, Maio - Junho, 2004

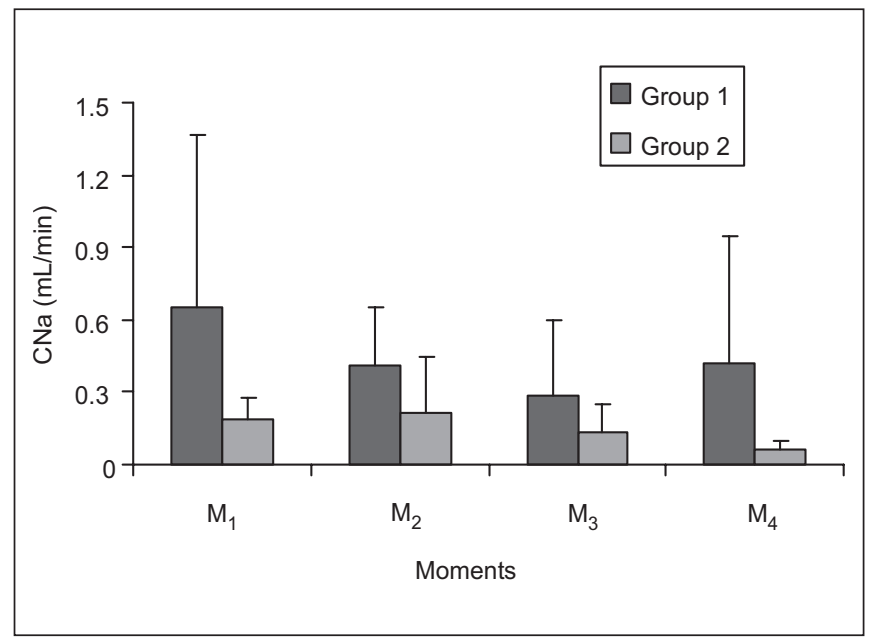

Figure 13 - Sodium Clearance. Mean and Standard Deviation in Both Experimental Groups

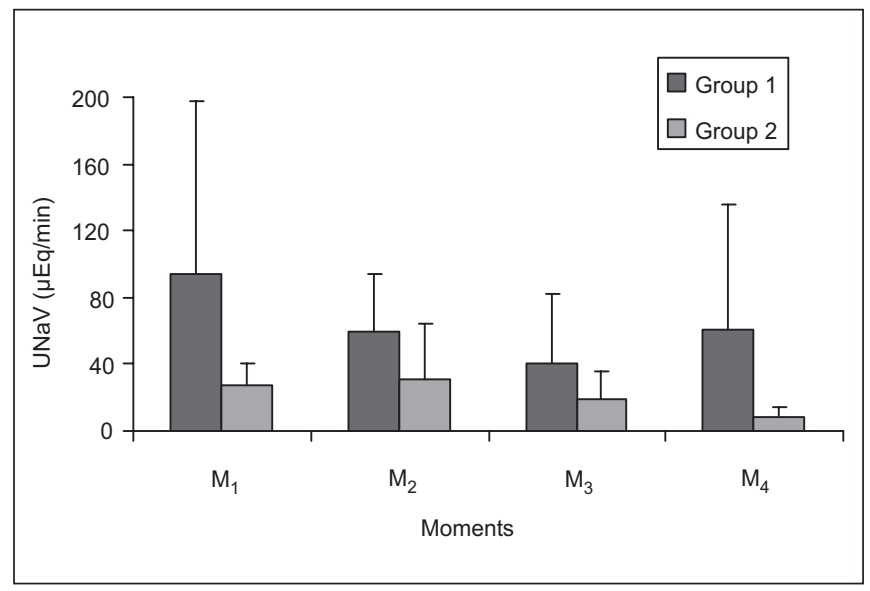

Figure 14 - Urinary Excretion. Mean and Standard Deviation in Both Experimental Groups

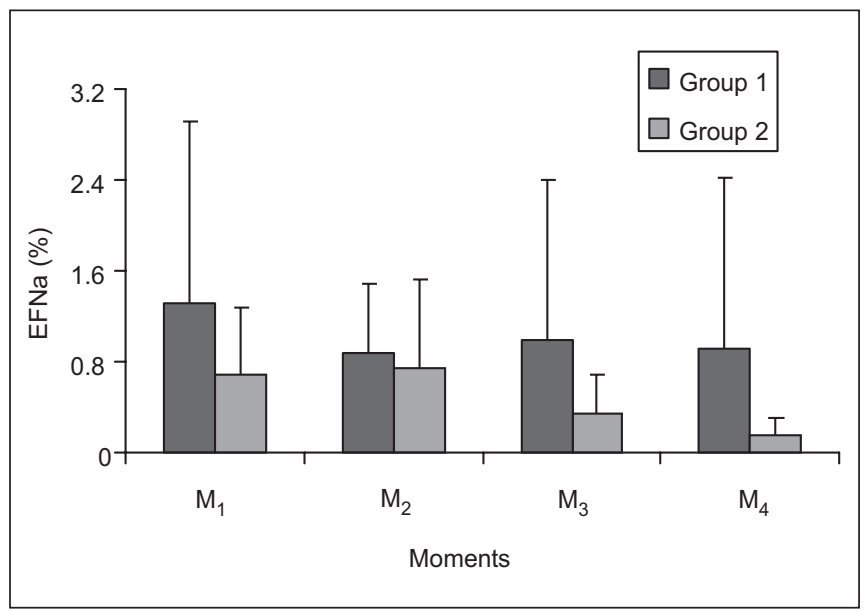

Figure 15 - Sodium Fractional Excretion. Mean and Standard Deviation in Both Experimental Groups 
Potassium clearance was similar for both groups with significant decrease in $\mathrm{M}_{2}$ and remaining like that until experiment completion. Group 1 values were significantly higher as compared to Group 2 (Figure 16).

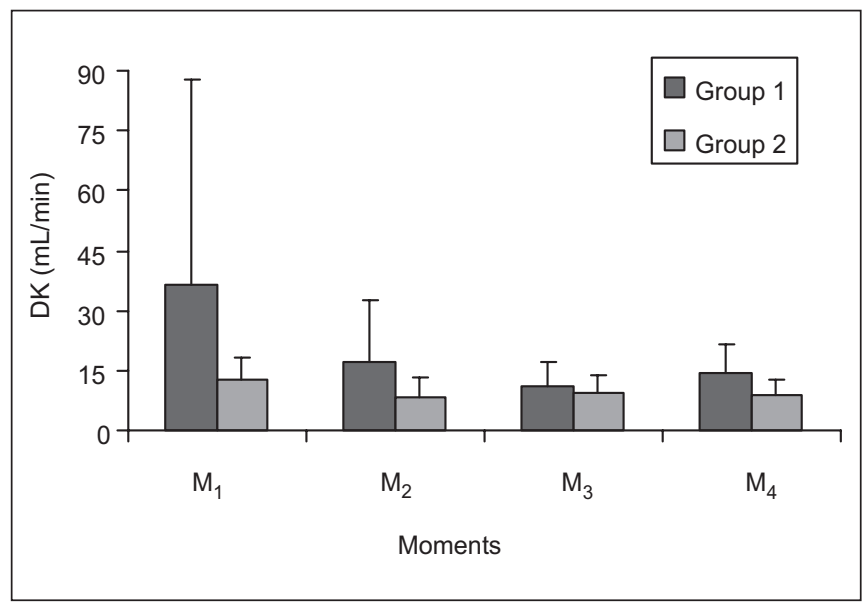

Figure 16 - Potassium Clearance. Mean and Standard Deviation in Both Experimental Groups

Potassium urinary excretion was similar for both groups and remained stable throughout the experiment. Group 1 values were significantly higher as compared to Group2 (Figure 17).

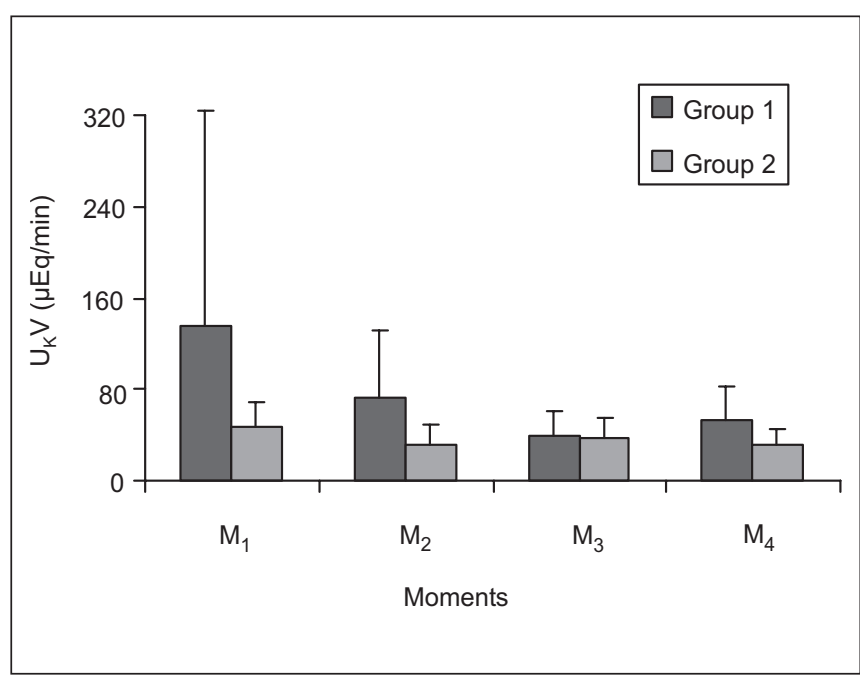

Figure 17 - Potassium Urinary Excretion. Mean and Standard Deviation in Both Experimental Groups

Potassium fractional excretion was similar for both groups and has decreased in $\mathrm{M}_{2}$ remaining like that until experiment completion (Figure 18).

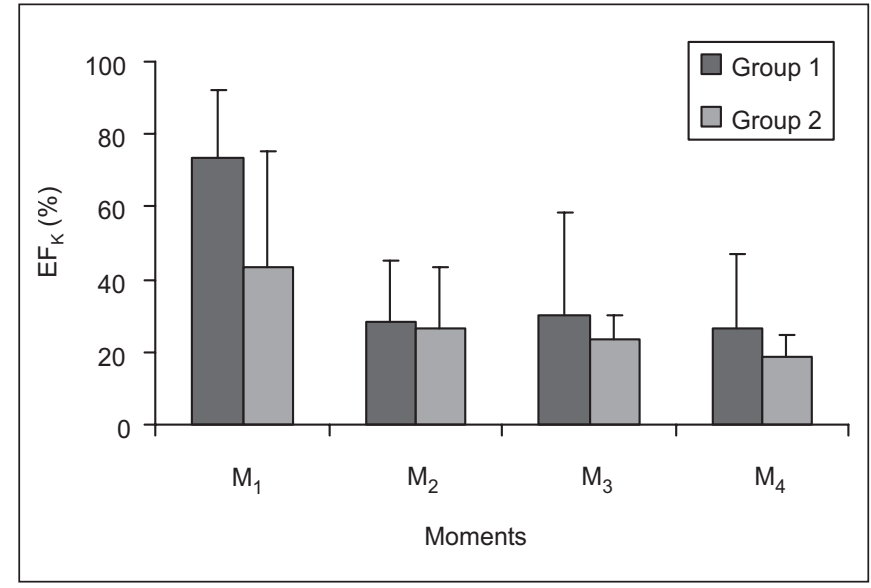

Figure 18 - Potassium Fractional Excretion. Mean and Standard Deviation in Both Experimental Groups

\section{DISCUSSION}

Pneumoperitoneum-induced intra-abdominal pressure (IAP) increase promotes systemic changes, the magnitude of which is directly related to IAP values used.

In patients with impaired renal function, be it acute or chronic, these may be major changes since there is often diuresis decrease during pneumoperitoneum.

Venous and arterial compression, added to parenchymal compression and hormonal changes, lead to decreased diuresis ${ }^{14}$. This is even observed in patients without any evidence of histological changes or renal tubular injury. In general, there is no diuresis and renal function impairment after peritoneal cavity deflation ${ }^{15}$.

An experimental study with dogs has observed $63 \%$ decrease in urinary output and $26 \%$ decrease in renal blood flow when kidneys were under $15 \mathrm{mmHg}$ IAP compression ${ }^{1}$. However, when IAP was above $20 \mathrm{mmHg}$, there has been $75 \%$ decrease in glomerular filtration and anuria was observed with $40 \mathrm{mmHg}$ IAP, values which have not changed with increased hydration ${ }^{2}$.

In a swine model submitted to different IAP values during 4 hours, it has been observed that pressures equal to or above $15 \mathrm{mmHg}$ have decreased cardiac output, urinary output, renal venous blood flow and creatinine clearance. These changes were not associated to permanent injuries or histological renal changes ${ }^{16}$.

Clinical and experimental studies on renal function and pneumoperitoneum are not uniform as to the volume of fluid to be administered, and vary 2 to $15 \mathrm{~mL} \cdot \mathrm{kg}^{-1} \cdot \mathrm{h}^{-1}$. Oliguria does not seem to be affected by hydration ${ }^{16-19}$.

In our study, animals have received $6 \mathrm{~mL} \cdot \mathrm{kg}^{-1} \cdot \mathrm{h}^{-1}$ hydration, aiming at keeping them hydrated and preventing hyper-hydration and dehydration.

High blood $\mathrm{CO}_{2}$ values may increase plasma rennin activity, plasma catecholamines, aldosterone and vasopressin concentrations during laparoscopic surgeries ${ }^{6,20,21}$. As a result 
of this hormonal stimulation there is renal vasoconstriction with decreased renal blood flow and glomerular filtration ${ }^{22}$.

The fast systemic vascular resistance increase after pneumoperitoneum has been correlated to hormonal changes with increased plasma rennin ${ }^{6,7,9}$ and vasopressin $3,18,23$ activity.

SR 4925 - a vasopressin antagonist - has inhibited systemic vascular resistance increase ${ }^{23}$. So, vasopressin has a major role in hemodynamic regulation during pneumoperitoneum 23,24

As to catecholamines, there is no temporal relationship between their plasma concentration increase and systemic vascular resistance and mean blood pressure increase ${ }^{24}$.

In our study, blood $\mathrm{CO}_{2}$ values and vasopressin concentrations have remained within physiological limits. Renal vascular resistance has not changed throughout the experiment for both groups, but Group 1 values were higher as compared to Group 2 and this fact was related to the characteristics of the studied group.

In favorable hemodynamic conditions there is increase in renal blood flow, renal plasma flow and filtration fraction with increased cardiac output and decreased peripheral resistance; in fact, there is a decrease in filtration fraction, that is, the rate between glomerular filtration rate and renal plasma flow, showing that increased renal plasma flow was proportionally higher that glomerular filtration rate ${ }^{25}$.

So, filtration fraction decrease observed in our study, where there has been cardiac index increase and systemic vascular resistance decrease ${ }^{26}$, would be a consequence of increased renal plasma flow.

In extracellular fluids, sodium cation and the two major anions following it, chloride and bicarbonate, are responsible for $90 \%$ solutes in this compartment ${ }^{25}$. So, it is possible to estimate plasma osmolality through sodium plasma concentrations.

Plasma osmolality remains virtually unchanged due to the integration hypothalamic-hypophysial-renal and antidiuretic hormone, which is critical for the maintenance of such stability ${ }^{27}$. Plasma sodium has not significantly changed in both groups throughout the experiment with stable plasma osmolality and vasopressin plasma values.

Positive pressure ventilation may promote cardiac output and blood pressure decrease in situations such as hypovolemia and increased intra-thoracic pressure, as it is the case with pneumoperitoneum ${ }^{28,29}$.

Cardiac output and blood pressure decrease leads to renal blood flow decrease, rennin-angiotensin-aldosterone system activation and higher sodium and water absorption, changing plasma osmolality.

Our study has not observed cardiac index, mean blood pressure ${ }^{26}$ and renal blood flow changes in both groups, thus not expecting influences in plasma osmolality, which has remained stable throughout the procedure and was not influenced by pneumoperitoneum, hydration or mechanical ventilation.

Most remarkable event triggered by IAP increase on kidneys is diuresis decrease ${ }^{1,8,17,19,30}$. It is related to vasopressin plasma concentration increase $e^{3,8,23}$ and to mechanical compression of renal parenchyma, renal artery, renal vein and inferior vena cava, triggering negative effects on renal blood flow and renal function.

Diuresis is increased when there is extracellular volume expansion ${ }^{25}$, natriuretic hormone release ${ }^{31}$, stretching receptors and atrial pressure stimulation ${ }^{32}$ and preload increase.

Mechanical ventilation, for determining intra-thoracic pressure increase and, as a consequence, decreased venous return and cardiac output, may stimulate vasopressin release and atrial natriuretic factor inhibition. Our study, however, has not observed cardiac index decrease ${ }^{26}$ or changes in plasma vasopressin values for both groups.

Vasopressin, which plays a major role in renal perfusion and water reabsorption has not changed in our study, and IAP has not exceeded $15 \mathrm{mmHg}$ for one hour. These factors, associated to our hydration pattern with maintenance of normovolemia may have contributed for the maintenance of diuresis throughout the procedure for both groups. Higher Group 1 values would be individual characteristics and in line with higher mean blood pressure values of this group.

Considering that there has been normovolemia, hemodynamic stability and no changes in plasma vasopressin values for both groups, it is possible to conclude that the lack of significant changes in plasma sodium, sodium clearance, sodium urinary excretion and sodium fractional excretion was a reflex of hemodynamic stability promoted by ventilatory modes.

It is known that structural polarity of proximal tubule cells is essential for the primary function of the selective reabsorption of molecules in the tubular fluid, and that it is changed during renal ischemia ${ }^{33}$. Changes in $\mathrm{Na}$, K-ATPase pump position are responsible for high sodium excretion fractions characteristic of the urine of patients with post-ischemia acute renal failure ${ }^{34}$.

It has to be highlighted that there has been no renal sodium excretion in any moment for both groups, but in analyzing absolute sodium clearance, sodium urinary excretion and sodium fractional excretion values it was possible to infer that there has been a trend toward a decrease in their values for both groups throughout the experiment, what adds to urinary osmolality decrease and free water clearance increase.

So, trends to sodium clearance and urinary and fractional excretion decrease could reveal mild renal function involvement triggered by IAP increase, leading to parenchymal compression and promoting possible changes in tubular absorption and secretion.

Higher values for these attributes in Group 1 could be a reflex of higher mean blood pressure values observed in $\mathrm{G} 1^{25,26}$.

As to potassium, it is believed that almost everything that is filtered is reabsorbed and that potassium excreted by the urine is originated from tubular secretion. When tubular fluid reaches the distal tubule, $85 \%$ to $95 \%$ of filtered potassium have already been absorbed and excreted concentrations and amounts in final urine have no major variations ${ }^{35}$.

Potassium excretion regulation is influenced by its plasma and cellular concentrations which, when increased, also act 
on adrenal aldosterone secretion, which stimulates sodium reabsorption and potassium excretion, without a mandatory coupling between these cell absorption and excretion mechanisms $^{36}$

Potassium secretion in the distal tubule may increase as a function of fluids overload and luminal flow increase ${ }^{36}$. Volume expansion is able to decrease plasma rennin activity with consequent decrease in aldosterone production and lower potassium excretion. Increased intratubular flow decreases potassium luminal concentration, favoring the passive ionic movement of the cell to the tubular lumen toward the ion gradient.

There is an inverse correlation between acid and potassium secretion in the acid-base balance. Alkaloid states lead to higher potassium excretion; as opposed, potassium excretion is decreased in acid states ${ }^{36}$. Plasma $\mathrm{pH}$ and potassium ratio is such that for each $0.1 \mathrm{pH}$ unit increase or decrease, there is $0.63 \mathrm{mEq} \cdot \mathrm{L}^{-1}$ plasma potassium decrease or increase.

Ventilatory modes used have determined $\mathrm{pH}$ values decrease, which was more evident in Group 1 and could peak with lower potassium clearance and fractional excretion.

Positive pressure ventilation may act on potassium through aldosterone's hormonal action, inducing higher sodium reabsorption and potassium excretion. This, however, was not observed since potassium fractional excretion has not increased, showing that ventilatory modes have not interfered with rennin-angiotensin-aldosterone system.

In a clinical trial with patients submitted to laparoscopic cholecystectomy, there have been no changes in potassium fractional excretion ${ }^{37}$. However, in swine submitted to pneumoperitoneum, increased potassium plasma concentrations were observed ${ }^{16,38}$. In both studies, plasma concentrations were below values potentially able to trigger arrhythmias.

Potassium serum concentrations have not changed in our study. However, potassium clearance and fractional excretion have decreased as from pneumoperitoneum, and potassium urinary excretion tended to decrease in both groups. Higher potassium clearance and fractional excretion in Group 1 was probably due to higher mean blood pressure levels in this group.

It is possible that there has been no aldosterone influence in potassium handling since there has been no increase in sodium reabsorption, which would lead to ion excretion. Since hemodynamic parameters have remained stable, animals' volume status was enough to not influence tubular flow.

Although potassium plasma concentrations have not changed, some factors, such as renal parenchymal compression determined by increased IAP and/or arterial $\mathrm{pH}$ decrease, may have contributed for lower ion clearance and fractional excretion observed in both groups as from $\mathrm{M}_{2}$, as a consequence of its higher reabsorption and/or lower secretion. Water transport is always secondary to other solutes transport by purely physical-chemical mechanisms. In most distal nephron segments, water transport is modulated by antidiuretic hormone or vasopressin ${ }^{39}$.
The ratio between solutes excretion and water may be studied considering that urinary volume is made up of "two waters" - one to contain all solutes (osmoles) excreted by urine and the other "water" to be excreted as such, pure and solute-free ${ }^{40}$.

Decreased urinary osmolality observed after pneumoperitoneum might have been a consequence of lower potassium ion clearance and excretion added to a trend to lower sodium ion clearance and excretion.

Higher free water clearance observed as from pneumoperitoneum is also in line with decreased urinary osmolality, representing the trend to higher and solute-free urinary filtrate. Vasopressin plasma levels have remained within normal limits, what excludes the participation of the hormone in increased free water clearance.

Osmolar clearance, corresponding to plasma volume $(\mathrm{mL})$ in which all osmotically active substances in the time unit are removed, was not significantly different between groups.

$\mathrm{PAH}$ clearance values maintenance throughout the procedure reflects stable glomerular filtration rate, thus excluding any renal function impairment secondary to pre-renal factors.

So, decreased urinary osmolality and increased free water clearance could be a consequence of renal parenchyma involvement as a consequence of IAP increase, which would compress kidneys and lead to changes in nephrons absorption and secretion ability.

So, it is possible to conclude from our experiment that, among different variables which could have determined changes in renal hemodynamics and function, ventilatory modes have not led to systemic changes able to determine renal function involvement. It could be inferred that renal parenchyma compression determined by increased intra-abdominal pressure could have been responsible for changes in renal potassium excretion.

\section{REFERÊNCIAS - REFERENCES}

01. Razvi HA, Fields D, Vargas JC et al - Oliguria during laparoscopic surgery: evidence for direct renal parenchymal compression as an etiologic factor. J Endourol, 1996;10:1-4.

02. Harman PK, Kron IL, Mclachlan HD et al - Elevated intra-abdominal pressure and renal function. Ann Surg, 1982;196:594-597.

03. Punnonen R, Viinamaki O - Vasopressin release during laparoscopy: role of increased intra-abdominal pressure. Lancet, 1982;1:175-176.

04. Joris JL, Noirot DP, Legrand MJ et al - Hemodynamic changes during laparoscopic cholecystectomy. Anesth Analg, 1993;76:1067-1071.

05. Ortega A, Peters J, Incarbone R et al - A prospective randomized comparison of the metabolic and stress hormonal responses of laparoscopic and open cholecystectomy. J Am Coll Surg, 1996;183:249-256.

06. Koivusalo AM, Kellokumpu I, Scheinin M et al - Randomized comparison of the neuroendocrine response to laparoscopic cholecystectomy using either conventional or abdominal wall lift techniques. Br J Surg, 1996;83:1532-1536. 
07. O'leary E, Hubbard K, Tormey W et al - Laparoscopic cholecystectomy: haemodynamic and neuroendocrine response after pneumoperitoneum and changes in position. $\mathrm{Br} \mathrm{J}$ Anaesth, 1997;76:640-644.

08. Koivusalo AM, Kellokumpu I, Ristkari S et al - Splanchnic and renal deterioration during and after laparoscopic cholecystectomy: a comparation of the carbon dioxide pneumoperitoneum and the abdominal wall lift method. Anesth Analg, 1997;85:886-891.

09. Koivusalo AM, Kellokumpu I, Scheinin M et al - A comparison of gasless mechanical and conventional carbon dioxide pneumoperitoneum methods for laparoscopic cholecystectomy. Anesth Analg, 1998;86:153-158.

10. Hamilton BD, Chow GK, Inman SR et al - Increased intra-abdominal pressure during pneumoperitoneum stimulates endothelin release in a canine model. J Endourol, 1998;12:193-197.

11. David CM - Ventilação Mecânica: Repercussões Hemodinâmicas, em: David C - Ventilação Mecânica. Rio de Janeiro, Revinter, 1996;77-86.

12. Gouvea F, Ferreira E, Campos AP et al - Monitorização hemodinâmica: métodos invasivos. Rev Bras Anestesiol, 1992;42:21-41.

13. Morrison DF - Multivariate Statistical Methods. New York: McGraw-Hill, 1967;338.

14. Dualé $\mathrm{C}$, Bolandard $\mathrm{F}$, Duband $\mathrm{P}$ et al - Conséquences physiopathologiques de la chirurgie coelioscopique. Ann Chir, 2001;126:508-514.

15. Dunn MD, McDougall EM - Renal physiology. Laparoscopic considerations. Urol Clin Norh Am, 2000;27:609-614.

16. McDougall EM, Monk TG, Wolf Jr JS et al - The effect of prolonged pneumoperitoneum on renal function in an animal model. J Am Coll Surg, 1996;182:317-328.

17. Chang DT, Kirsch AJ, Sawczuk IS - Oliguria during laparoscopic surgery. J Endourol, 1994;8:349-352.

18. Dolgor B, Kitano S, Yoshida T et al - Vasopressin antagonist improves renal function in a rat model of pneumoperitoneum. $\mathrm{J}$ Surg Res, 1998;79:109-114.

19. London ET, Ho HS, Neuhaus AM et al - Effect of intravascular volume expansion on renal function during prolonged $\mathrm{CO}_{2}$ pneumoperitoneum. Ann Surg, 2000;231:195-201.

20. Walder AD, Aitkenhead AR - Role of vasopressin in the haemodynamic response to laparoscopic cholecystectomy. $\mathrm{Br} \mathrm{J}$ Anaesth, 1997;78:264-266.

21. Corwin C, Fabrega AJ, Scott-Conner C - Neurohormonal Response to Laparoscopy and Acute Rise in Intra-Abdominal Pressure, em: Rosenthal RJ, Friedman RL, Phillips EH - The Pathophysiology of Pneumoperitoneum. Berlin, Springer, 1998;99-113.

22. Diebel LN - Renal Function and Circulation under the Influence of Pneumoperitoneum, em: Rosenthal RJ, Friedman RL, Phillips EH - The Pathophysiology of Pneumoperitoneum. Berlin. Springer, 1998;62-69.

23. Mann C, Boccara G, Pouzeratte $Y$ et al - The relationship among carbon dioxide pneumoperitoneum, vasopressin release, and hemodynamic changes. Anesth Analg, 1999;89:278-283.

24. Joris JL, Chiche JD, Canivet JL et al - Hemodynamic changes induced by laparoscopy and their endocrine correlates: effects of clonidine. J Am Coll Cardiol, 1998:32:1389-1396.

25. Marcondes M - Regulação do Volume de Fluido Extracelular, em: Malnic G, Marcondes M - Fisiologia renal, $3^{\mathrm{a}} \mathrm{Ed}$, São Paulo: EPU, 1986a;253-270.

26. Almeida AV, Ganem EM, Carraretto AR et al - Alterações hemodinâmicas durante o pneumoperitônio em cães ventilados com volume e pressão controlados. Rev Bras Anestesiol, 2003:53:756-766
27. Marcondes M - Alterações do Metabolismo da Água, em: Malnic G, Marcondes M - Fisiologia renal. $3^{\text {a }}$ Ed, São Paulo: EPU, 1986b;271-82.

28. Fessler HE, Brower RG, Wise RA et al - Effects of positive end-expiratory pressure on the gradient for venous return. Am Rev Respir Dis, 1991;143:19-24.

29. Kotanidou A, Armaganidis A, Zakynthinos S et al - Changes in thoracopulmonary compliance and hemodynamic effect of positive end-expiratory pressure in patients with or without heart failure. J Crit Care Med, 1997;12:101-111.

30. Cisek LJ, Gobet RM, Peters CA - Pneumoperitoneum produces reversible renal dysfunction in animals with normal and chronically reduced renal function. J Endourol, 1998;12:95-100.

31. Rahman SN, Batt, AT, Dubose TD et al - Differentiating clinical effects of ANP in oliguric and non-oliguric ATN. J Am Soc Nephrol, 1995;6:474-475.

32. Moe GM, LegaulT L, Skorechi KL - Control of Extracelular Fluid Volume and Phatophysiology of Edema Formation, em: Brenner BM, Rector FC - The Kidney, $4^{\text {th }}$ Ed, Philadelphia: WB Saunders, 1991;623-676.

33. Chen J, Doctor B, Mandel MJ - Cytoskeletal dissociation of ezrin during renal anoxia. Role in microvillar injury. Am J Physiol, 1994;36:784-795.

34. Edelstein $\mathrm{CL}$, Ling $\mathrm{H}$, Schrier RW - The nature of renal cell injury. Kidney Int, 1997:51:1341-1351.

35. Aires MM - Reabsorção e Secreção Tubular: Técnicas de Depuração, em: Malnic G, Marcondes M - Fisiologia Renal, $3^{\text {a }}$ Ed, São Paulo: EPU, 1986;89-111.

36. Furtado MR - Balanço do Potássio e sua Regulação, em: Malnic G, Marcondes M - Fisiologia Renal, $3^{\mathrm{a}}$ Ed, São Paulo: EPU, 1986;299-310.

37. Iwase $\mathrm{K}$, Takenaka H, Ishizaka T et al - Serial changes in renal function during laparoscopic cholecystectomy. Eur Surg Res, 1993;25:203-212.

38. Pearson MR, Sander ML - Hyperkalaemia associated with prolonged insufflation of carbon dioxide into the peritoneal cavity. Br J Anaesth, 1994;72:602-604.

39. Malnic G - Excreção Renal de Água e Eletrólitos, em: Malnic G, Marcondes M - Fisiologia Renal, $3^{\text {a }}$ Ed, São Paulo: EPU, 1986a;125-156.

40. Malnic G - Concentração e Diluição Urinária, em: Malnic G, Marcondes M - Fisiologia Renal, $3^{\text {a }}$ Ed, São Paulo: EPU, 1986b;173-213.

\section{RESUMEN}

Almeida AV, Ganem EN - Efectos del Pneumoperitonio sobre la Hemodinámica y Función Renal de Perros Ventilados con Volumen y Presión Controlados

JUSTIFICATIVA Y OBJETIVOS: No existen estudios que asocien los efectos determinados por las modalidades ventilatorias a las repercusiones renales durante el pneumoperitonio. El objetivo de este trabajo fue evaluar las alteraciones en la hemodinámica y función renal determinadas por el pneumoperitonio en perros con ventilación a volumen y presión controlados.

MÉTODO: Dieciséis perros anestesiados con tiopental sódico y fentanil fueron divididos en Grupo 1, volumen controlado y Grupo 2, presión controlada y sometidos a pneumoperitonio de 10 y $15 \mathrm{mmHg}$. Fueron estudiados flujo sanguíneo renal, resistencia vascular renal, depuración de para-aminohipurato de sodio, sodio plasmático, potasio plasmático, osmolalidad plasmática, depuración de creatinina, fracción de filtración, volumen urinario, osmolalidad urinaria, depuración osmolar, 
depuración de agua libre, depuración de sodio, excreción urinaria de sodio, excreción fraccionaria de sodio, depuración de potasio, excreción urinaria de potasio, excreción fraccionaria de potasio. Los datos fueron colectados en 4 momentos. $M_{1}$, antes del pneumoperitonio; $M_{2}, 30$ minutos después pneumoperitonio con $10 \mathrm{mmHg} ; M_{3}, 30$ minutos después pneumoperitonio con $15 \mathrm{mmHg} ; \mathrm{M}_{4}, 30$ minutos después de la deflación del pneumoperitonio.
RESULTADOS: Las depuraciones de para-aminohipurato de sodio y creatinina permanecieron constantes en ambos grupos durante el experimento. Los valores plasmáticos del sodio y del potasio no se alteraron. Ocurrió diminución a partir de $M_{2}$ de la depuración y de la excreción fraccionaria de potasio en ambos grupos.

CONCLUSIONES: Las modalidades ventilatórias no determinaron diferencias en la hemodinámica renal entre los grupos estudiados. El pneumoperitonio, ocasionando compresión del parenquima renal, puede tener determinado alteraciones en la reabsorción y/o secreción del potasio. 